(Aus der deutschen Universitätskinderklinik in der böhmischen Landesfindelanstalt in Prag [Vorstand: Prof. R. Fischl].)

\title{
Beitrag zur Kenntnis der Nierenerkrankungen bei kongenitaler Lues.
}

\author{
Von \\ Dr. Max Frank, \\ Assistent der Kllnik. \\ Mit 6 Abbildungen im Text. \\ (Eingegangen am 22. Juni 1922.)
}

Es ist natürlich, daß die Erbsyphilis bei den mannigfaltigen Erscheinungen, die sie an den Organen des mit ihr behafteten Individuums hervorruft, auch die Nieren nicht verschont. Bemerkenswert ist es, daß die Erforschung der Pathologie der Niere bei kongenitaler Lues erst zu einem verhältnismäßig späten Zeitpunkte einsetzte. Dieser Umstand wird dadurch verständlich, daß die bei angeborener Syphilis bestehenden Nierenveränderungen dem Beobachter bei makroskopischer Untersuchung in der größten Anzahl der Fälle verborgen bleiben. In der Regel macht dieses Organ makroskopisch den Eindruck des normalen. Erst die mikroskopische Untersuchung gestattet, die dort vorhandenen pathologischen Prozesse zu erkennen. Nur fortgeschrittene gummöse Prozesse, wie sie in den Nieren kongenital-syphilitischer Kinder in einer geringen Anzahl von Fällen vorkommen, überzeugen schon bei makroskopischer Betrachtung von der Erkrankung dieses Organes. Dies ist bei anderen Organen nicht der Fall. So imponieren z. B. schon die Leber und die Milz durch ihre Volumsvergrößerung als pathologisch verändert. Daher kam es auch, daß, während für die damalige Zeit außerordentlich bedeutungsvolle Abhandlungen über die Erkrankungen verschiedener Organe bei Erbsyphilis längst bestanden, die Pathologie dẹr Nieren, abgesehen von kleineren nebenhin gemachten Bemerkungen, erst recht spät in die Literatur eintrat. Erst 1891 besprach Stroebe in einer umfangreicheren Abhandlung diesen Gegenstand.

Er setzte sich zuerst mit der Frage auseinander, ob es überhaupt, abgesehen natürlich von echten gummösen Prozessen, spezifische luetische Erkrankungen der Niere gebe. Darüber war damals noch kein entscheidendes Urteil gefällt worden. Stroebe beschrieb zwei kongenital-luetische Fülle, von denen nur das Alter des ersten - der Foetus stand im 10. Lunarmonat - angegeben wurde. 
Bezüglich der Nieren sei folgendes aus dieser Arbeit hervorgehoben: Es fand sich in diesen Organen eine reichliche Vermehrung des interstitiellen Bindegewebes, das besonders in der Umgebung der kleinen Arterien infiltriert war. An diesen Infiltrationen waren hauptsächlich .Leukocyten beteiligt. Eine viel größere Bedeutung als diesen Infiltrationsherden maB der Autor aber jenen epithelialen Bildungen bei, die sich als embryonales Nierenparenchym erwiesen. In den Nieren eines ausgetragenen Neugeborenen, die als Kontrollpräparate dienten, fanden sich keinerlei derartige embryonale Bildungen vor. Als Grund für das Zurückgebliebensein in der Entwicklung der Niere wurde der durch das syphilitische Gift auf das Bindegewebe ausgeübte Reiz, der letzteres zur Wucherung veranlassen sollte, angesehen. Diese Wucherung sollte wiederum das Parenchym in seiner Ausdehnungsfähigkeit behindern. Utberdies dürfte wahrscheinlich auch eine direkte Giftwirkung auf das Parenchym vorhanden gewesen sein. Als Stütze für diese Anschauung verwies Stroebe auf die Untersuchungsergebnisse Riedels. Dieser fand, da $B$ beim Menschen die Neubildung von Glomerulis und geschlängelten Kanälehen zur Zeit der Geburt nicht mehr stattfindet, erwähnt aber auch die Angaben von Toldt, nach dessen Untersuchungen eine Neubildung dieser Gewebsbestandteile auch noch in den ersten Tagen des extrauterinen Lebens fortgesetzt würde. Einige Jahre später beschäftigte sich Hochsinger eingehend mit dem Problem der kongenitalen Nierensyphilis. Seine Studien über die Niere bei dieser Krankheit schlossen sich denen Stroebes an. In den Nieren zweier kongenitalluetischer Säuglinge, von denen der eine 3, der andere 6 Wochen alt war, fand er deutliche Bilder unfertiger Entwicklung des Nierenparenchyms sowie entzündliche Prozesse des Interstitiums und der Glomeruli. Als Ort, an dem sowohl die Entwicklungshemmungen als auch die entzündlichen Prozesse am stärksten vorhanden waren, wurde die subcapsuläre (neogene) Zone angegeben. Die Entwicklungshesnmung bestand in folgendem: Die Glomeruli waren in viel geringerer Anzahl, als es der Norm entsprochen hätte, vorhanden. Dort, wo sie vorhanden waren. waren sie viel kleiner, oder sie präsentierten sich wie in Stroebes Fällen als unfertige Gebilde. Die sie bildenden Zellen hatten embryonalen Charakter. Die Entwicklung der Glomeruli in der neogenen Zone war zumeist in dem Stadium, in welchem das periphere Ende der Nierenkanälchen halbmondförmige Gestalt annimmt. Von der für das spätere Leben charakteristischen Gefäßverteilung daselbst war noch nichts zu finden. Als verhältnismäßig reifste Form waren jene Glomeruli zu betrachten, die schon in ihrem Innern bereits Gefäßknäuel bargen, Als Fehlbildungen sieht Hochsinger gewisse schlauchförmige mit Cylinderepithel ausgekleidete Hohlräume an, die voneinander und von der Umgebung durch interstitielles Gewebe getrennt waren. Er glaubte, daß es sich hier um abgeschnürte Teile von NierenkanäIchen handelte. Die entzündlichen Verände. rungen des Interstitiums bestanden in Infiltrationen. Die Epithelien der Harnkanälchen waren fettig degeneriert. Die Bowmanschen Kapseln beherbergten exsudative Entzündungsprodukte. Die Kerne der Capillaren waren gewuchert. Auf Grund dessen gelangte Hochsinger zu der Ansicht, da.B die Entwicklungshemmungen für die Nieren kongenital-luetischer Säuglinge spezifisch wären, und daß die Syphilis allein imstande wäre, eine Nephritis zu erzeugen.

In demselben Jahre, in dem die Arbeit Hochsingers erschien, veröffentlichte R. Hecker seine erste Abhandlung über kongenitale Lues. Bezüglich der Veränderungen der Niere kam der Autor zu folgendem Schlusse. Bei sämtlichen Fällen von Syphilis sowie auch dort, wo zwar der klinische Befund an Lues denken ließ, das Ergebnis der Sektion keine sicheren Anhaltspunkte dafür ergab, fanden sich Veränderungen in den Nidren, so daß die mikroskopische Untersuchung dieses Organs für die Diagnose ausschlaggebend war. Bei den luetischen Frühgeburten, die Hecker untersuchte, war immer eine kleinzellige Infiltration der 
Wandung oder nächsten Umgebung der kleinsten Rindengefäße zu finden. Nicht immer konnte die Auswanderung von Leukocyten in das Innere der Boumanschen Kapsel ebenso wie die Proliferation des Kapselepithels festgestellt werden. Wandverdickungen der GefäBe kamen nur zweimal vor. Bei ausgetragenen Kindern traten die Infiltrationen in den Hintergrund, und degenerative Vorgänge am Epithel beherrschten das Bild. Hochgradiger wurden diese Veränderungen bei Kindern, die bereits einige Wochen gelebt hatten. Für direkt spezifisch hielt Hecker nur die zuerst genannten Infiltrate. Neben diesen schrieb er auch den schon beschriebenen Zeichen von Entwicklungshemmung diagnostische Bedeutung zu.

Eine andersartige Stellung nahm Karvonen zur kongenitalen Nierensyphilis ein. Er bestätigte zwar die früher gemachten histologischen Befunde, maB ihnen aber eine andere Bedeutung bei. Seine Ausführungen gipfelten in der Anschauung, daß viele bisher als entzündlich gedeutete Erscheinungen als Zeichen einer unvollendeten Entwicklung direkt durch das sypjhilitische Virus bedingt anzusehen wären. Die von anderen Autoren als kleinzellige Infiltration bezeichnete Erscheinung, hielt Karvonen meist für normal, indem er der Meinung Ausdruck gab, daB nur der Kernreichtum des embryonalen Gewebes einen entzündlichen ProzeB vortäuschte. Dort, wo er entzündliche Veränderungen neben Entwicklungshemmungen zugab, $\mathrm{maB}$ er den entzündlichen Veränderungen geringere und sekundäre Bedeutung bei. Damit stellte er sich in Gegensatz zu Hochsinger und Stroebe. Für diagnostisch äußerst wichtig hielt Karvonen das Vorhandensein embryonalen Gewebes in der subcapsulären Zone. Bedeutungsvoll erschien ihm auch der Umstand, daß er bei genauer Zählung der übereinanderliegenden Glomeruli zu dem Resultate kam, daß die Anzahl derselben bei kongenital-luetischen Früchten eine geringere als bei normalen wäre. Auch diese Tatsache wurde als eine Entwicklungshemmung gedeutet. Aus dem Vorhandensein unfertiger Gewebsbildungen in der neogenen Zone, dem Orte, an dem das Wachstum der Niere in der Fötalzeit vor sich geht, schloß Karvonen auf einen zu verhältnismäßig später Zeit embryonaler Entwicklung stattfindenden Angriff des Syphilistoxins auf den jungen Organismus. Für ein weiteres Zeichen der Unreife, das charakteristisch für syphilitische Neugeborene wäre, hielt Karvonen das Vorhandensein breiterer als der Norm entsprechender Bindegewebsstränge in der Rindenzone. Ebensolche Bedeutung wurde auch den in der Rindenzone häufig anzutreffenden Cysten zugeschrieben. Erscheinungen geweblicher Entwicklungshemmungen sollten die auch von diesem Autor beschriebenen kavernösen Bluträume in der subcapsulären Zone darstellen. Häufigeres Vorkommen von entzündlichen Prozessen gab Karvonen nur bei Säuglingen zu. Immerhin schienen ihm aber auch hier die Hemmungsbildungen des Nierenparenchyms bedeutungsvoller zu sein, insofern als besonders sie für die kongenitale Lues charakteristisch wären. Unter den entzündlichen Bildungen nahmen wiederum die interstitiellen Prozesse die Stelle vor den im Nierenparenchym bzw. in den Glomerulis ein.

Die hier genannten Ansichten Karvonens blieben nicht unwidersprochen. 1902 bezeichnete Hecker in einer weiteren Arbeit, in der er die Nieren wiederum als besonders geeignetes Organ zur Bestätigung der Diagnose Lues congenita bei abgestorbenen Früchten hinstellte, die interstitiellen und besonders perivasculären Zellanhäufungen als entzündliche Produkte. Daneben stellte er als wichtiges Zeichen endarteritische und entziundliche Vorgänge im Parenchym hin, die aber erst im Säuglingsalter eine gewisse Bedeutung erhaiten dürften. In der Frage der Bedeutung der Entwicklungshemmungen änderte Hecker seine Meinung. Nur die Tatsache der verminderten Zahl der übereinanderliegenden Glomeruli ließ er gelten und glaubte auch an eine toxisch bedingte Proliferation des mesenchymalen Gowebes. 
Von anderer Seite hingegen wurden die Untersuchungsergebnisse Karvonens bestätigt. Störck fand häufig Entwicklungsanomalien an den Glomerulis bei Erbsyphilis. Das Vorhandensein der neogenen Zone konnte Störck in diesen Fällen noch einige Monate nach der Geburt beobachten. Sie waren allerdings im Säuglingsalter seltener als beim Neugeborenen. Auch auf das verhältnismäBig häufige Vorhandensein von Cysten wurde hingewiesen. Nicht unähnlich lauteten die Untersuchungsergebnisse von $\mathrm{Cassel}$, der Gelegenheit hatte, 31 kongenital-luetische Säuglinge zu untersuchen. Als charakteristisch für die Niere bei kongenitaler Syphilis bezeichnete Cassel interstitielle und periadventitielle Wucherungen neben cystischer Degeneration der Glomeruli. Außerdem kämen bei Föten noch Zeichen von Entwicklungshemmung dazu, wie sie schon beschrieben wurden.

Die hier beschriebenen Unstimmigkeiten bezüglich der Pathologie der Nieren bei kongenitaler Lues veranlaßten $H a h n$, das vorhandene Material nochmals zu sichten bzw. einige eigene Fälle zu besprechen. Hahn kam zu folgenden Ergebnissen. Er unterschied zwischen fötaler und infantiler Nierensyphilis. Bezüglich der fötalen syphilitischen Nierenerkrankungen schloß sich der Autor der Meinung Karvonens, Heckers und Finkelsteins an, daß diffus auftretende zellige Infiltrationen ebenso wie Zellanhäufungen in der subcorticalen Zone nur in Verbindung mit anderen spezifischen Erscheinungen diagnostisch für Lues verwertbar wären. Bezüglich der diagnostischen Beurteilung der neogenen Zone meinte er, da $B$ sie beim ausgetragenen Kinde, möglicherweise auch sohon beim 9 und 10 Monate alten Foetus wohl den Verdacht, jedoch nicht die Diagnose der Lues rechtfertigen würden. Die infantile Nierensyphilis teilte $H a h n$ 1. in die akute und subakute interstitielle, 2 . in die chronische interstitielle und 3 . in die akute parenchymatöse und hämorrhagische Nephritis ein. Die Fälle der zweiten und dritten Gruppe waren sehr spärlich und, wie selbst der Autor zugibt, keineswegs durchwegs einwandfrei. Von diesen soll noch an späterer Stelle die Rede sein.

Schließlich sei noch der Meinung Aschoffs Raum gegeben, der den diagno. stischen Wert eines längeren Bestehens der neogenen Zone gering einschätzte und mehr die interstitiellen kleinzelligen Infiltrate wertete, die sich teils aus fötalen Blutbildungsherden, teils aus den von Ceelen beschriebenen Plasmazellhaufen zusammensetzen sollten.

Wir haben im vorstehenden nur die größeren Arbeiten, die sich mit unserem Gegenstande beschäftigen, genannt. Aus ihnen geht hervor, daß die Pathologie der Nieren bei kongenitaler Syphilis keineswegs endgültig geklärt erscheint, weshalb wir uns entschlossen haben, diese Frage neuerlich zu bearbeiten. Wir möchten das Material in drei Gruppen einteilen, und zwar: 1. Neugeborene, 2. unbehandelte und 3. behandelte Säuglinge. Dazu sei bemerkt, daß wir als Titel der Gruppe 1 den Namen Neugeborene gewählt haben, weil wir dortselbst die Nieren solcher Kinder besprechen, die lebend zur Welt kamen, wenn sie auch öfters nur ganz kurze Zeit am Leben geblieben sind. Manche würden wohl gewisse Fälle dieser Gruppe, deren Tebenszeit nur nach Stunden zählte, zur Gruppe der fötalen Nierensyphilis rechnen. Wir wollten diesen Ausdruck hier deshalb vermeiden, weil ja bei kongenital-luetischen Früchten der Eintritt einer Frühgeburt mehr oder weniger zur Regel gehört und selbst Individuen des ersten 'Säuglingsalters öfters weit davon entfernt sind, die. Charakteristica des ausgetragenen Säuglings aufzuweisen. 
Daß natülich die pathologischen Veränderungen der clsten Gruple ofters den für fötale laes charalintistischen gleichen, soll dadureh

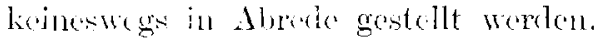

Einiges soll hice noth iiber die Methodik der histologivehen Lutcrsuchung gesagt werden. Es wurden sehnitte der Nion teils nach den gewöhlichen Methoden acturbt, teils nach Levediti mit Silber imprëgniert. Den rom Funonen als wathenatisebe Wethode der cilomervluxzilhlung benannten Forgang haben wir nicht beructsichtigt. Kartonen fand, wie schon bemerlat. dak die Zahl der senkrecht übereinander liegenden Glomeruli beim syphilitischen Kinde Tskiner als beim normalen wäte. Zur Zählung der Glomenuli arbeitete er cisce Methode aus. Sie wurde

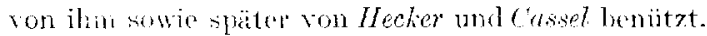
Da sich aber nach don leröfentlichungen der genannten Autoren oft nur undeutliche Enterschiede zwischen den Xieren normaler und luetischer Föten zeigten und überdies dir von den cinzehnen Autoren gefundenen Zablenwerte weit auseinanderlacen, so haben wir von dieser llethode abgesehen. Wir geben dafür hier ain Beispicl aus der Literatur an.

\section{Giruppe 1 .}

Fall 1. Find der M. M. Mutter 23 Jahre alt, 1916 spentangeburt, Kind o Honate alt gestorben, 1917 Aussehlag an ganzen Köper, der 4 Wochen andaunt, 1921 zweite Geburt. WaR. positiv.

Kind $2400 \mathrm{~g}$ schwer. $50 \mathrm{~cm}$ lang. Kam asphyttiseh zur Welt und starb nach 15. Min.

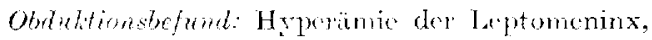
fast volstandige Atelektase der Lungen. Osteochondritis luctica. Die Nieren zeigten makroskopisch keine Verinderungert.

Wiliroshopiseh: Dientzindlichen Voünderungen, recheinen in gleicher Heise auf die Pindenzonen beider Nieren verteilt. Sie betreffen ausschlie Bliek das interstiticlle Geweb)e. Auch die Glomeruli weisen nicht dis seringsten entzinclichen Verïnderungen auf. Sie zeigen in dor periplearn Rindenzone be zijglich ihrer Entwirklung wrscherlen weit fortgosehrittene Stadien. Wan sieht bewinteude Glomerulusbildung, bej der cha periphere Ende der primären kanälehen sich

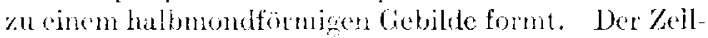
belay ist noch hochzylindrixch, die Zellkeme länglich

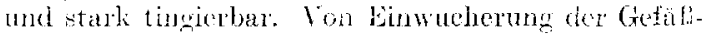
schlingen ist an vieled Sitellen noel licine sipur vor. hanken. Dameben sicht man weiter in ihrer Entwirklung fortgesehriteme (klomeruli. Die sielle, an? 


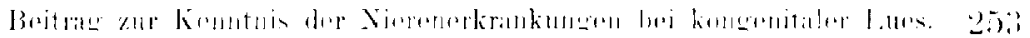

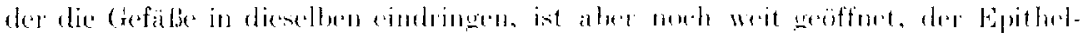

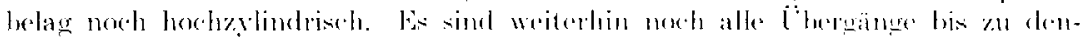

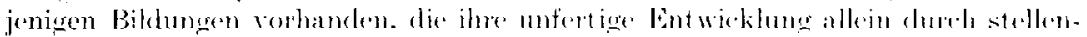

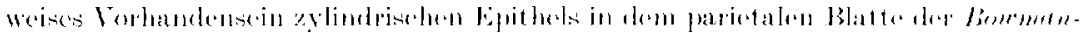

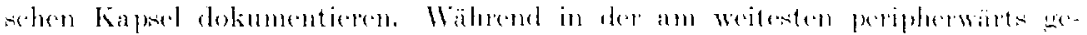

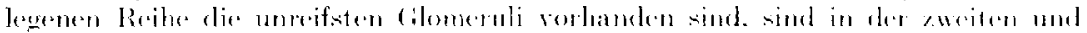

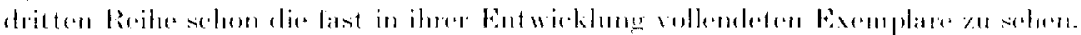

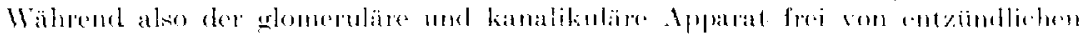

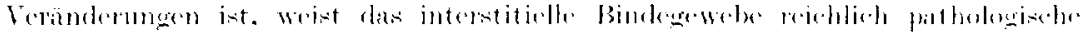

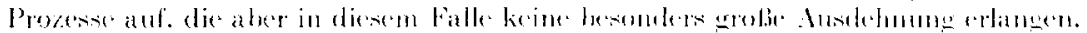

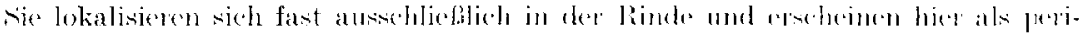

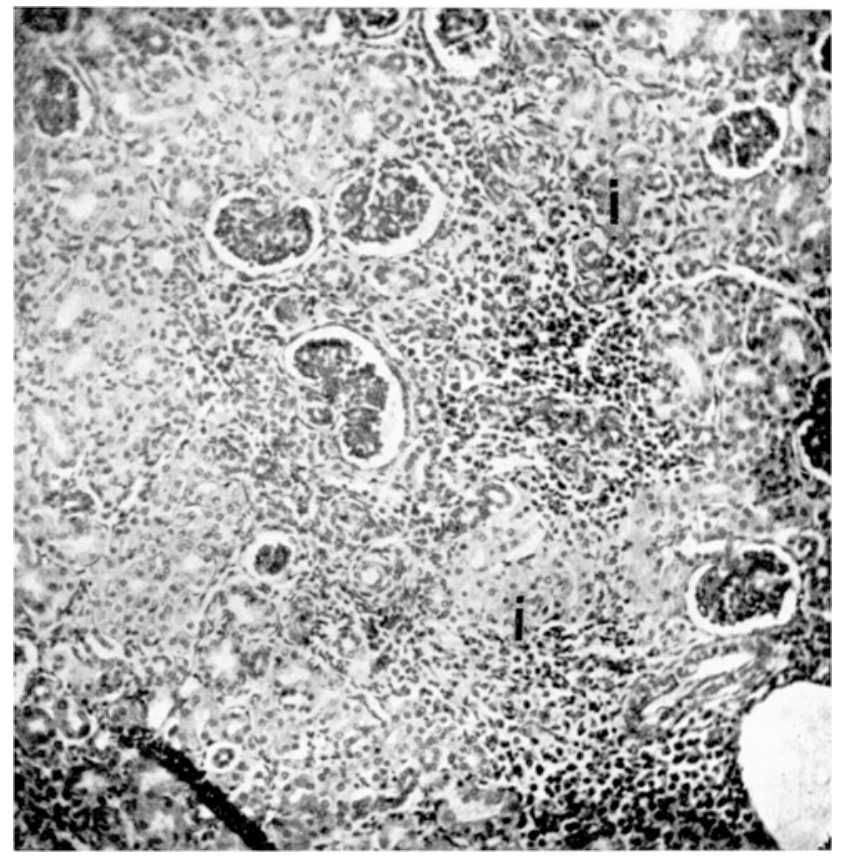

Ali. 1 .

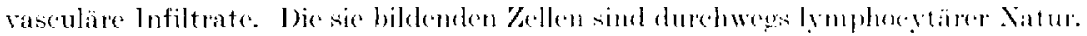

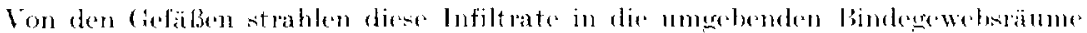

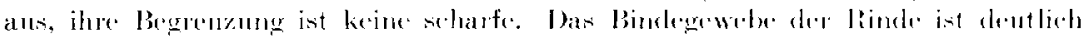

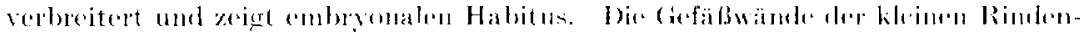

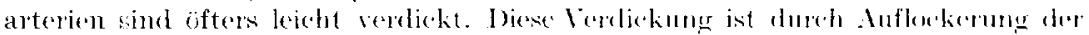

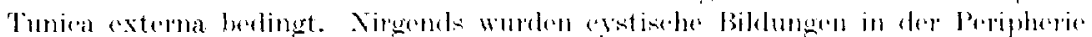

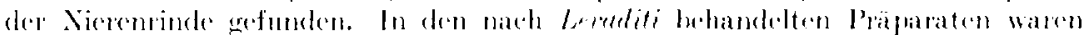

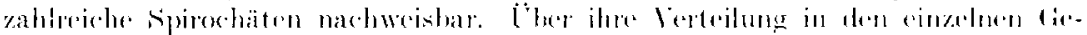

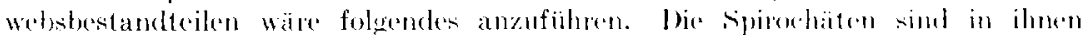

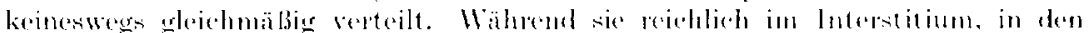

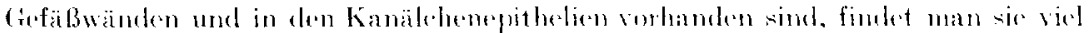

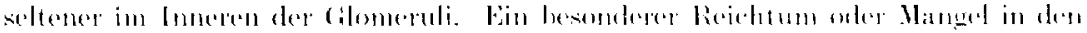

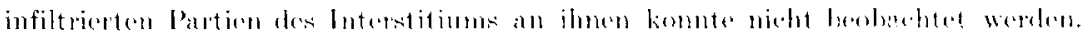




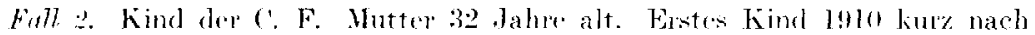

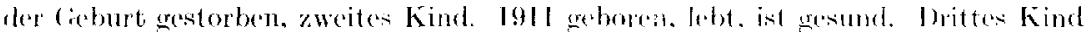

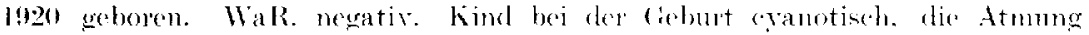

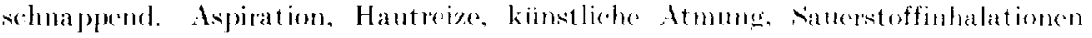

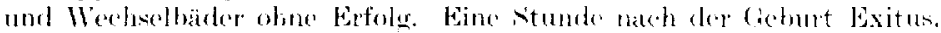

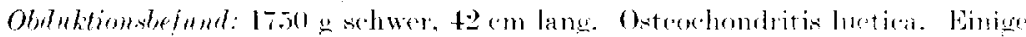

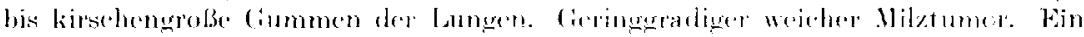

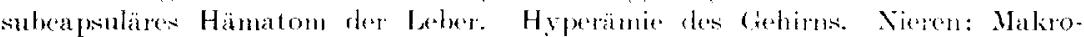
sokpische ohne. leränderume.

Mikroskopisch: Die Niren sind in weitem Ausmalie reänderte Doch haben diese veränderungen mehr herelfomieren Chatakter als in reston Falle.

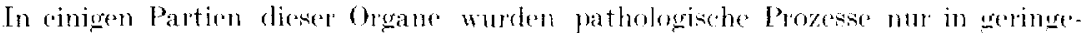

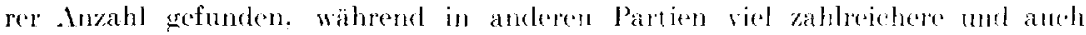

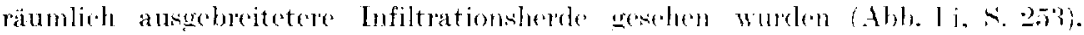
div stellenweise miteinander konflueden. An den stellen. wo uroblele Infiltrate

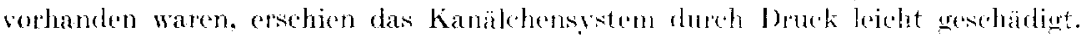

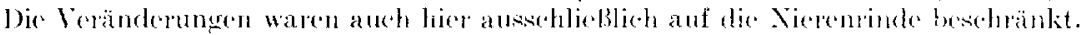

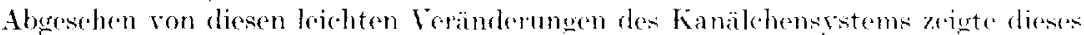

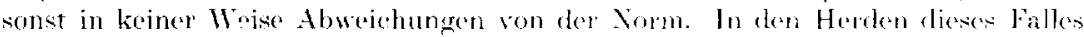
wah man neben Lymphoreten hänfig auch Plasmazellen sowie Epitheloidzellen und stellenweise mohr ader weniger revehlich junges Bindegewebe. Im Zent lum derselben befand sich der Durchsehnitt cines Gofabes, das öfters cine vorticlite

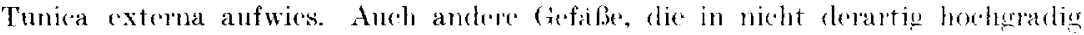
infiltrierter Umgebung lagen, zeigten Verdickung ihrer Wand und aufgelockerte lunica externa. In ihrer Entwicklung unfertige Glomeruli fand man bei diesem Falk auch noch in eingen weiter zontral grelegenen fehichten, als bei fall 1. Ebenso war auch hier das interstitielle fewebe ron deutlich embryonalem Cha-

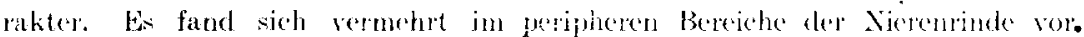

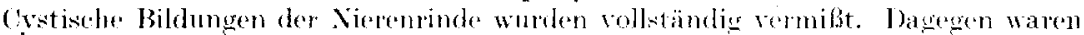

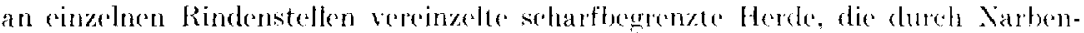
gewebe charakterisiert waren, zn schen. In den mit silber imprägnicrton Bräparaten wurde dic Spirochacta pallida nirerends irefunden.

Fall 3. Kind der P. K. Mutter: Kondylome in der Lngebung des Anus. WaR. positiv. Kind 2300 schwer, 42 con lang. 'Typische Fiuhgeburt. Pemphigus lucticus. Sattelnase. Reichliche Aspiration ron Prubtwasser, das teilweise rük. aspiriert werden konnte. Stirbt nach 25 Min.

Obduktionsbefund: I'neumonia alba mit melueren z. 'T'. verkästen (iummen. Fast vollständige Atelektase der lungen. Diffuse Hepatitis. Osteochondritis luetica. Ansgehreiteter Prmphigus lueticus. Odem des Gehirns. Siere: Makroskopisch ohne Veränderungen.

Mikroskopiseh: Auch in diesen Nieren finden sich deutliche pathologische Voränderungen. Allerdings sind sie nicht in so ausgebreptetem Mabe vorhanden. Auch hier zeigt das Kanälchersystem keine Abnomitäton. Ebenso sind auch hier embryonale Glomerulusbildungen in den periphersten Rindenschichten vorhanden. Sie bieten dasselbe Bild wie in den vorangchenden Fälen dar. Vereinzelte kernarme, als Narben zu bezeichnende kleinere Fleckchen finden sich in der peripheren Rindenzone. Die kleinen Arterien sinel mäBicr verdickt. Ias Gewebe ihrer peripheren Schichten ist ofters leicht aufgelockert und von lymphocytären Zellen mäBig stark infiltriert. Das interstitielle Bindegewebe ist ieichlich in der Rindenzone vorhanden. Die interstitiellen Infiltrate gruppieren sich um die Gefäbe und ragen manchnal zwisehen die Nierenkanälohen hincin. Öfter überschreitet die Größs derselben kaum die eines Glomerulus. Der Aufbau derselben 


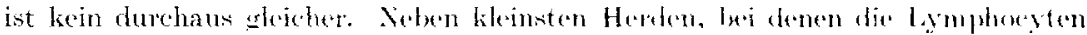

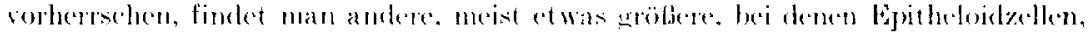

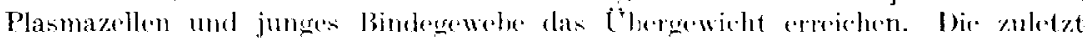
beschriebenen Herde erscheinen infelge der relativen ('hromatinarmut ihres keme.

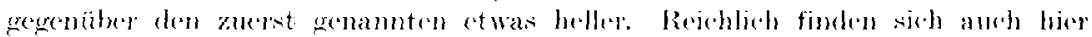

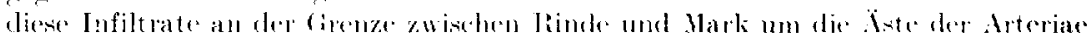

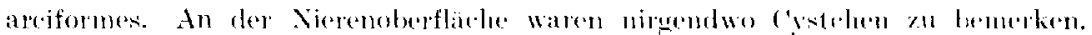
In den Levaditipräparaten wuden keine spirochäten gefundell.

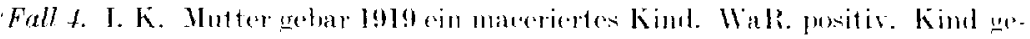

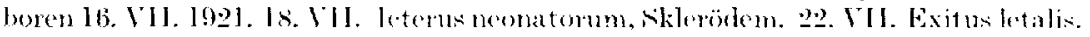

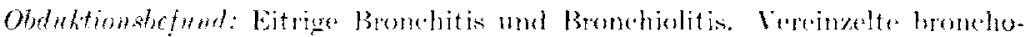

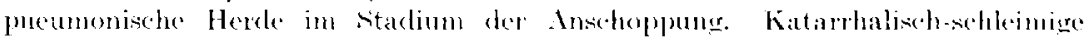

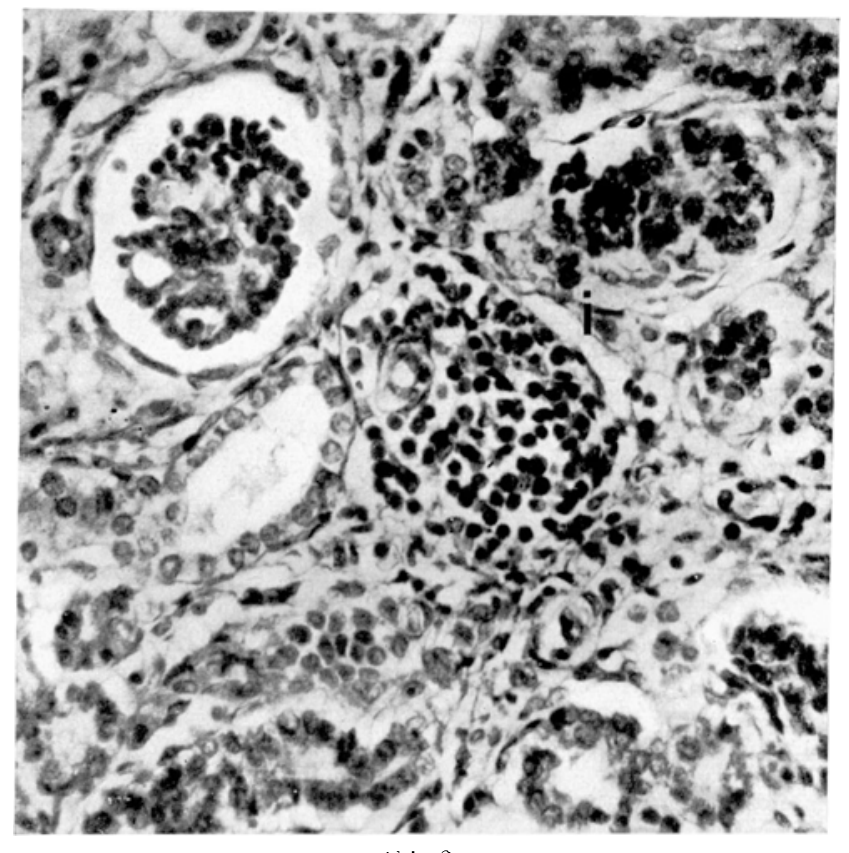

Hil. 2.

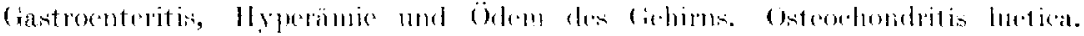
Milztumor. Die Nieren makroskepisell nommal.

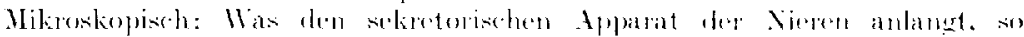

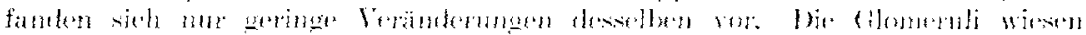

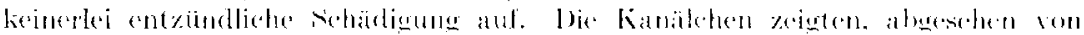

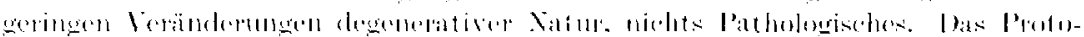

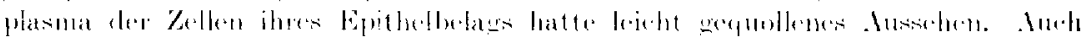

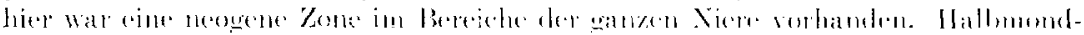

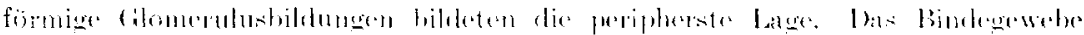

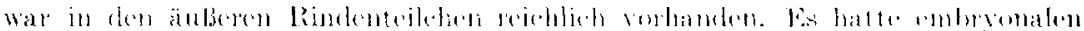

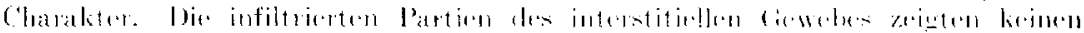

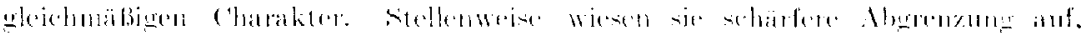

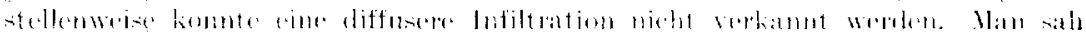




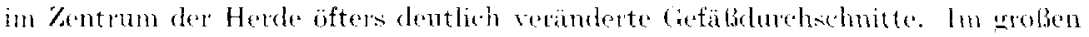
und ganzen zeigten auch hier dir Gefäbe Verdickungen ihrer Handung. bestingt

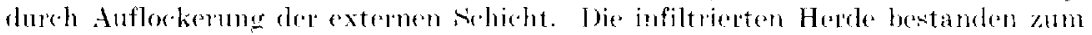

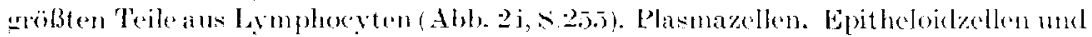
Bindegewebe waren nur sche spatich vorhanden. ('ysten im peripheren Rinden-

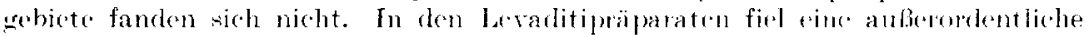

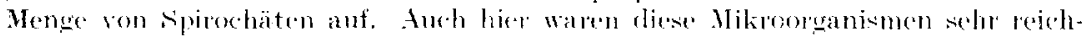
lich in den kanälchenwandungen vorhanden und kamen auch im Lumen dex-

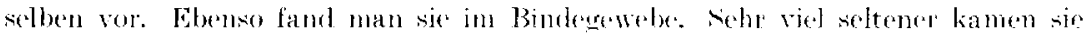
in den chomerulis, the zum gröBten Toil äberhaupt frei von spirochäten waren, vor. Auch in den pathologisch reranderten Partien des Interstitiums waren sie in ererineserer Zahl vorhanden.

Auschliebend an die Fälle dere ersten Gruple möchten wir die Ergebnisise der histologischen Lntersuchung der Nieren einiger sicher nicht: luetiachen Individuen der gleichen Altersstufe anführen, die zur Futscbediung darïber dienen sollen. Welche von den beschriebenen teränderungen als pathologisch anzusehen siul.

Fall 1. R. B. 4 Tage alte's Kind. 46 am lang, 2401) gehwer. MaR. der Mutter nerativ. Weder Hutter noch Kind zeigen irgendweldhe Zejeleen ron Lues.

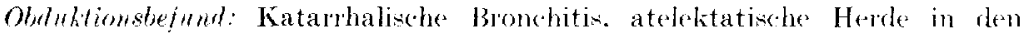
Lungen mit bromehopneumonischen Herden im Bereiche der Atclektasen. Knorpelknochengrenze normal. Nieren makroskopisch obne Veränderungenen.

Mikroskopiseh sind keinerlei Veränderungen an kanikchensystem wahrnehmbar. Was die Glomeruli anbelangt. so zeigen sie in rliesem Falle dieselben Zeichen nicht vollendeter. Entwicklung. wio diex bej den kongenital- luctischess

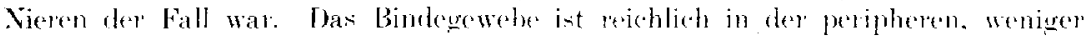
in der zentralen Rindengesshichte wohhandern. Xirgends finded man Ze-llanhäufungen im Interstitium.

Foll :. H. I. Kind, das 1 stunde gelelst hat. 48 cm lang. 25019 sthwer. Wak. der Mutter newativ. Werler bei Hutter noch Kind sind Zeichen ron Laess rorbanden.

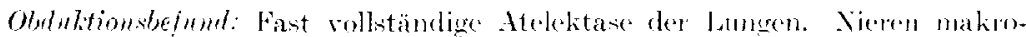
skopisch ohne loränderungen.

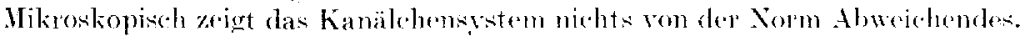
Die CHomeruli sind bis a tuf rereinzelte. Exemplare. deren Kapselepithel kubische Form anfweist, normal. Fie sind zumeist won der Peripherie bereits etwas in die

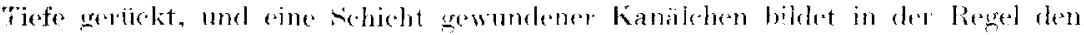
Abschlub des Nierenparenchyms.

Fall 3. K. M. Kind, das lo Stundengelebt hat. Be+ Mutter und Kind fehlt jecles Zejohen von Lues. WaR, eler Blutter negativ.

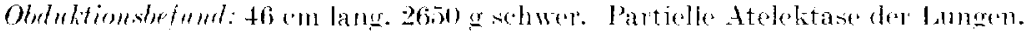
Offenes Fonamen wale. Fieren makenkopiech ohne Veränderungen.

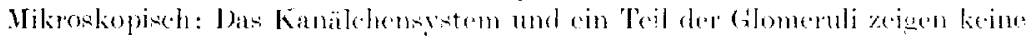

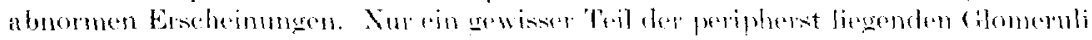

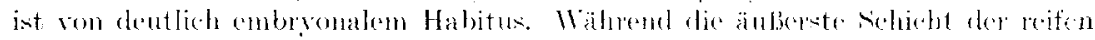

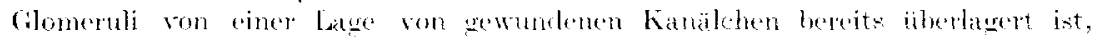

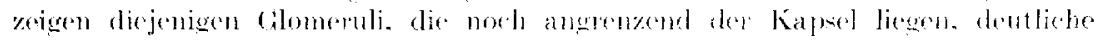
Zejehen unvollendeter Entwiekhug. Gntro ihnen fundet man Zellanhäufungen,

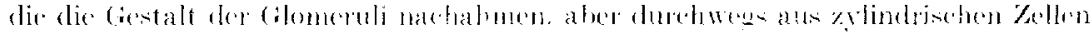

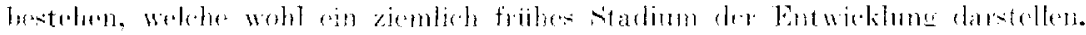




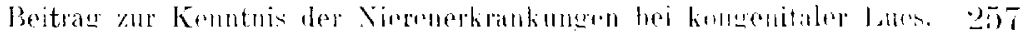

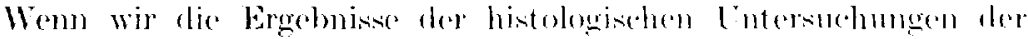

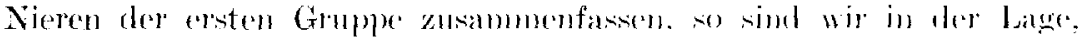

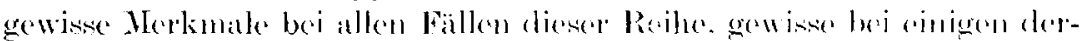

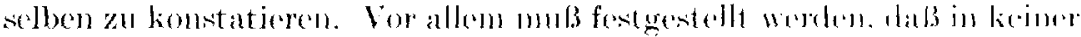

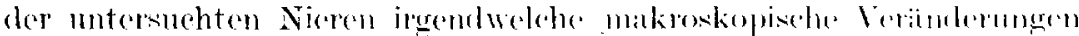

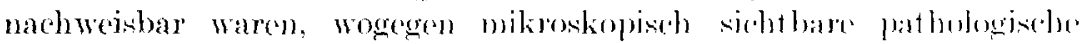

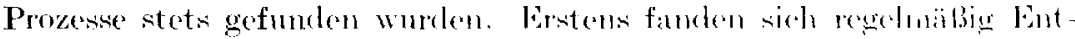

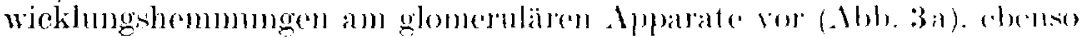

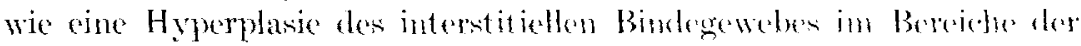

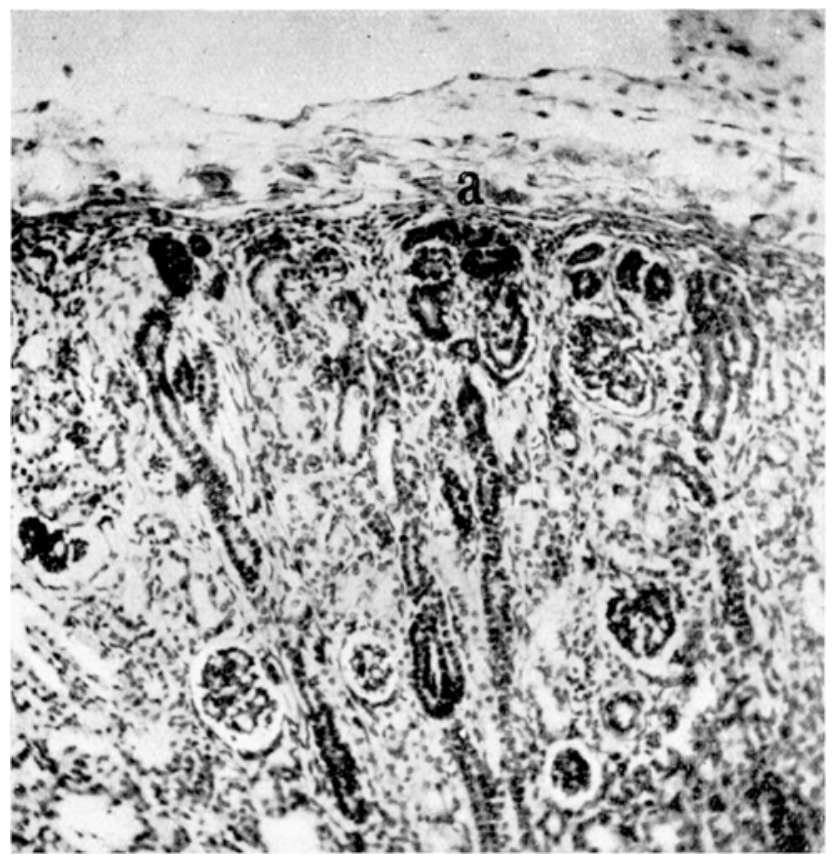

Al, , : :.

Nierenrinde. Zaweitens konnten regolmäbigr chmoniseh-entzündliehe

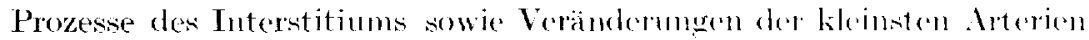
fextgestellt werken. Dritlens waren sipirochaten in zurei rom den be-

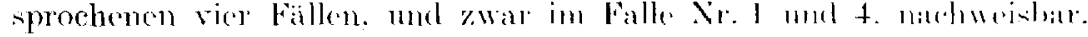

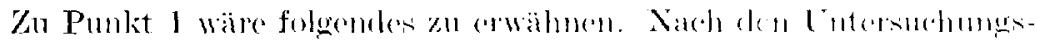

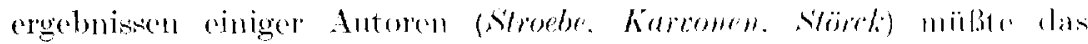

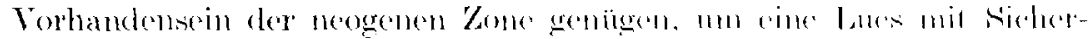

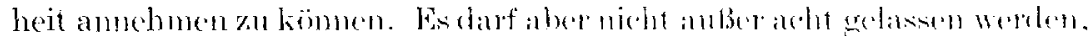

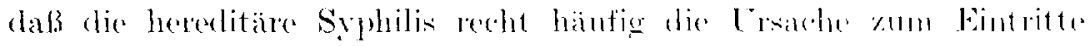

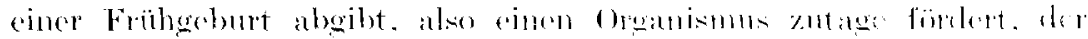

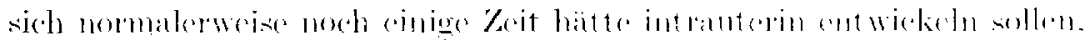


bevor er den Reifegrad crlangt hätte. anf Grund dessen eine normale

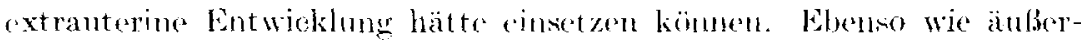
liche Merkmale das frühgeborene Individumm leicht crkmonen lassen, so muls ja dies auch bei den immeren Organen der Fall soin. Fes wollte deshalb gar nicht wundernehmen. wemn auch die Entwicklung inneres Organc bei frühgeburten nicht abgeschlossent ist. Auf die hier besprochenen Fälle ïbertragen wïrde dies bedeuten, dab es nur als otwas gan\% Natütiches anzusehen ist, wenn bei lebend geborenen luetischen Frühgeburten dine neogene Pindenzone rorhanden ist. Die Falle

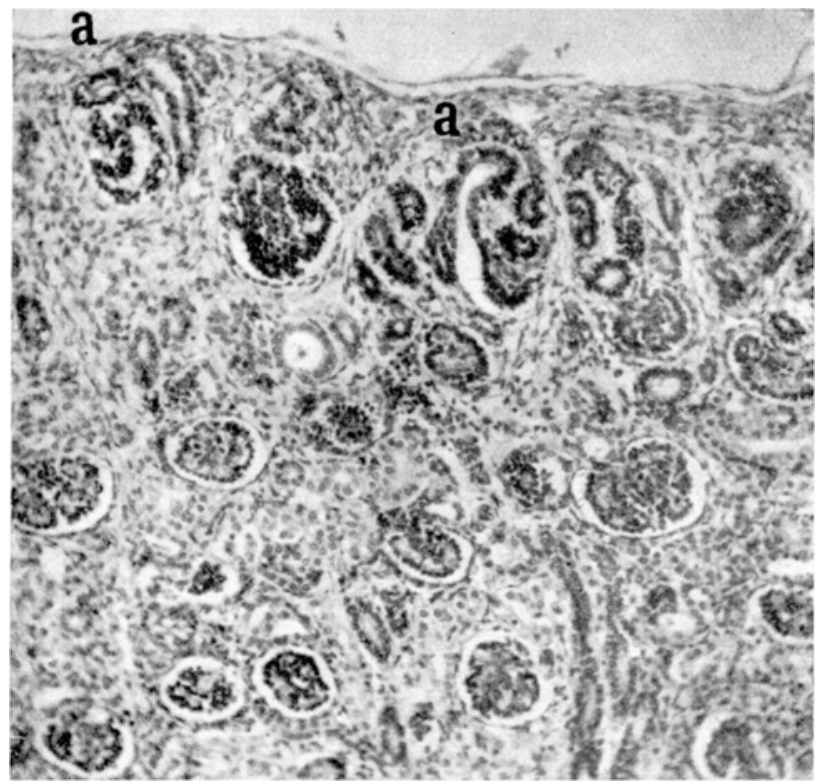

Ably. 1.

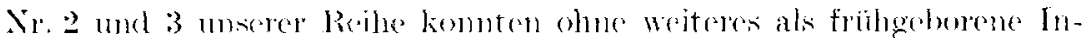
dividuen bezoichnet werden. Bei ihnen entfïllt also von rornherein die Terwertbarkeit des Merkmales der nicht abgeschlossenen Futwicklung des glomernlären Apparates als Hilfsmittel für die J)iagnose Later.

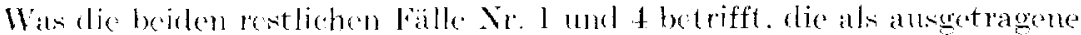

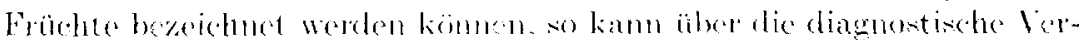

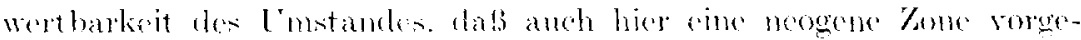
funden womke !nu an der Hand ron momalen Kontrollpriparaten

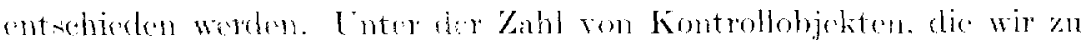

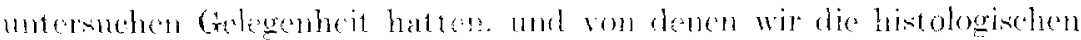

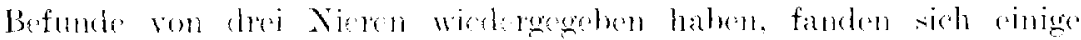

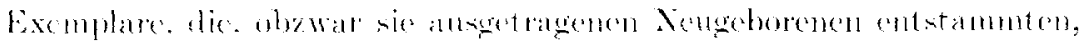




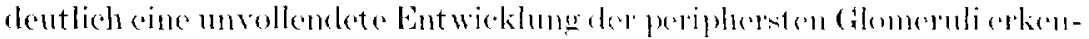

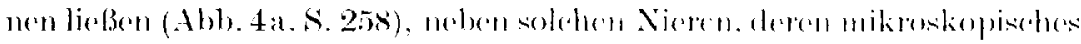

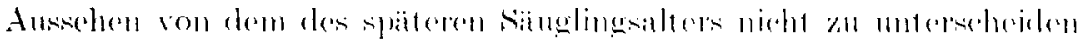

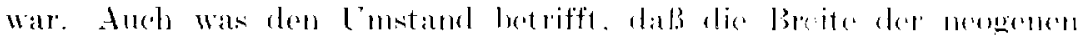

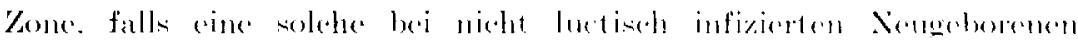

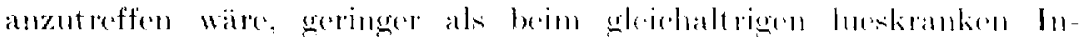

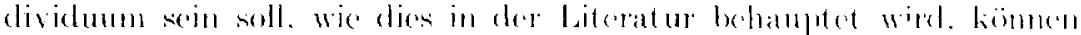

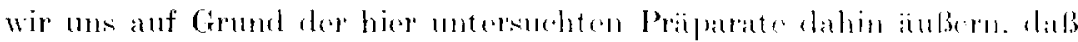

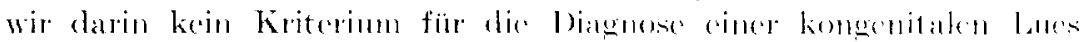

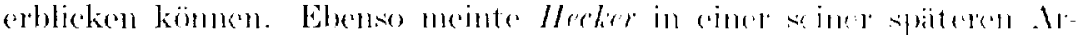

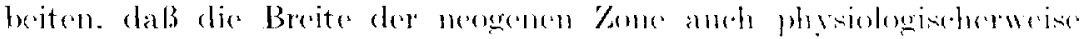

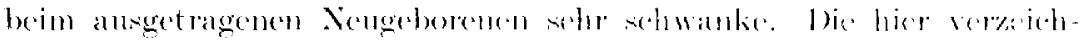

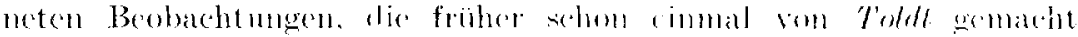

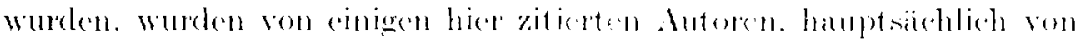

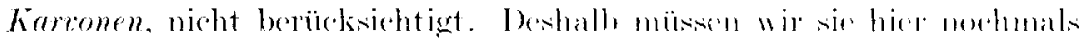

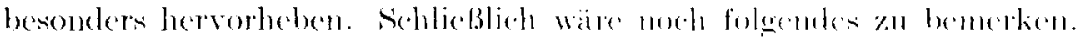

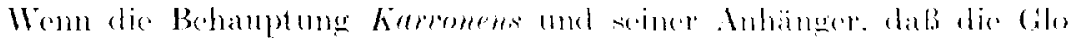

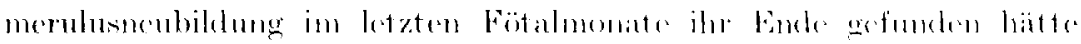

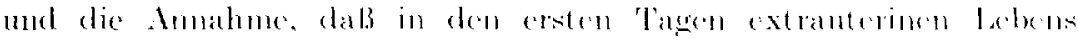

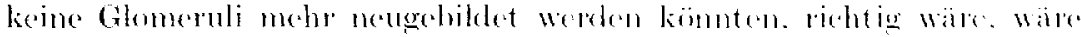

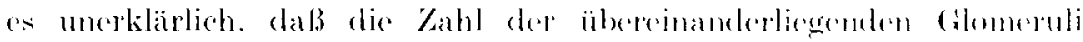

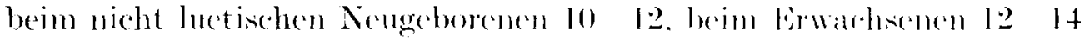

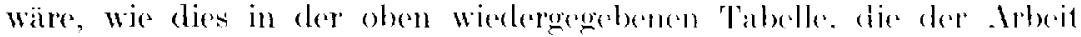
Hahns entnommen ist, festgestellt wirel.

Ebenso wie die Erseheimungen der Entwicklungshemmmug am glomerulären Apparat ist anch das bosombers in reep peripheren Rimdan-

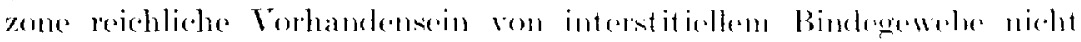
diagnostisch für hereditare Syphilis remolethar. Fe kam obenso nur als ein zeichen unvollendeter Entwieklumg der Nicren, wie sie ande beim Nengeborenen, der iuberlich die Zerichen der Reife triggt, nicht zul selten ist. angesehen werden.

Als Folge dieser Frgebnisse müssent wir dahed besomders betomen, daß es nicht angïngige ist, auf Grund des Vorhandenseins von embryonalen Glomerulusbiklungen, sowic anf Grund ron reichlich rorhandenen interstitidlem Bindegewebe von mehr oller wenige? embryenalem Charakter im Berejehe der peripheren Rimlenzone in der Niere eines Neugeborenen die I)iagnose cince kongenitalen Syphilis zu stellen. Fs erscheint uns auch nicht eimmal dic Ansicht Hahos zu Recht zu bestehen, die dahin geht, dab der Nabehweis der neogenen Zome. insbesondere aber eine Verbreiterung derselben. zum mindesten beim alusgetragemen Kinde, möglicherweise auch schon beim ! oker lo Honate alten Footus, wohl den Verdacht, nicht aber die Diagnose kongenitaler Lues rechtfertigte. 
Regelmäßig fanden sich herdförmige Prozesse im interstitiellen Bindegewebe. Die in der Literatur niedergelegten Meinungen über die Bedeutung derselben gehen auseinander. Während Karvonen diesen Bildungen zumeist einen entzündlichen Charakter abspricht, werden sie von Hecker und manchen späteren Autoren als entzündlich angesehen, eine Ansicht, der wir uns auf Grund des Vergleiches mit normalen Präparaten, in. denen diese interstitiellen Wucherungsvorgänge vollständig fehlen, anschließen müssen. Im Gegensatz zu Karvonen, der entzündlichen Infiltraten in der Niere kongenital-luetischer Früchte, von denen er meint, daß ihr Vorkommen daselbst überhaupt ein seltenes wäre, nur geringe Bedeutung beimißt, müssen wir diesen Bildungen, nachdem wir in der Lage waren sie bei Neugeborenen kongenitalluetischen Kindern regelmäßig zu finden, größte Bedeutung für die Diagnose einer kongenitalen Lues beimessen, zumal es sich ergeben hat, daß die Entwicklungshemmung des glomerulären Apparates für diese Diagnose nicht verwertbar ist. Die entzündlichen Produkte konnten wir in den einzelnen untersuchten Fällen in verschiedenen Stadien ihres Entwicklungsganges antreffen. Utber die Bedeutung des Vorhandenseins älterer oder jüngerer Stadien soll an einer späteren Stelle noch die Rede sein. Während die jüngeren Stadien sich dadurch kenntlich machten, daß sie infolge eines relativen Mangels an Bindegewebselementen ein lockeres Gefüge zeigten und durch das überwiegende Vorhandensein von Lymphocyten ein charakteristisches Aussehen erlangten, waren die älteren Prozesse infolge der Gegenwart zahlreicher Plasmazellen, Bindegewebselemente und auch epitheloider Zellen gekennzeichnet. Die jüngeren Herde zeigten auch keine so scharfe Begrenzung gegen das gesunde Gewebe wie die älteren. Außerdem war an den letzteren eine weiter vorgeschrittene Veränderung an den Gefäßen wahrzunehmen. Es wäre noch zu bemerken, daß in keinem dieser Herde nekrotische Stellen gesichtet werden konnten. Neben diesen hier näher beschriebenen Herden fanden sich in den Präparaten der Fälle Nr. 2 und 3 an der Rindenperipherie einige Stellen, die als Narben angesprochen werden mußten und die wir nicht anders wie als Endstadien der hier genannten interstitiellen Entzündungsprozesse auffassen konnten.

Die Gefäßveränderungen waren ziemlich verbreitet und regelmäßig in allen untersuchten luetischen Nieren vorhanden. Sie beschränkten sich auf die kleinsten zwischen den Kanälchen verlaufenden Arterienäste. Gefäßveränderungen bei kongenitaler Lues wurden schon früher beschrieben. Spanudis fand sie in den Lungen, Marchiafava, Stroebe, Karvonen, Hecker, Massalongho und andere in den Nieren. Auch in anderen Organen kongenital-luetischer Säuglinge wurde ihr Vorhandensein beobachtet. Hutinel und Hudelo machten auf dieselben in der 
Beitrag zur Kenntnis der Nierenerkrankungen bei kongenitaler Lues. 261

Leber aufmerksam. Es handelte sich dabei größtenteils um das Ergriffensein der äußeren Gefäßhaut. An den Gefäßen des Darmes wurden derartige Prozesse von Mraček gefunden. Ebenso wie Mraček im Darm konnten wir in den Nieren die kleinen Arterien im Bereiche der infiltrierten Herde am meisten verändert finden. In allen vier Fällen war regelmäßig die Tunica externa pathologisch verändert. Es zeigte sich daselbst eine Verdickung und stellenweise Auflockerung ihrer äußersten Wandschichten. Öfters konnte auch eine mäßig starke lymphocytäre Infiltration konstatiert werden. Obzwar nun die Tunica intima der Gefäße in allen Fällen unverändert war, so ist aus den Präparaten unserer Nieren ersichtlich, daß diejenigen, die in ihrem Interstitium ältere Entzündungsprozesse aufwiesen, auch durch deutliche Auflockerung und Infiltration der Tunica externa der kleinsten Arteriolen, die im Bereich der infiltrierten Herde verliefen, gekennzeichnet waren. Immerhin erscheinen die Veränderungen an den Gefäßen, verglichen mit den ausgedehnten und oft schweren Prozessen des Interstitiums, als sehr geringfügig. Auf Grund der vorliegenden Tatsachen $\mathrm{mu} B$ zwischen den Veränderungen der mittelgroßen Arterien, wie sie sich zum Beispiel in der Heubner schen Endarteritis der Basilararterien des Gehirns äußern, und den Prozessen, die sich in gewissen kleinsten im Parenchym verlaufenden Arterien abspielen, streng geschieden werden. Bilder, wie sie Hecker an den kleinsten arteriellen Gefäßen in Organen von kongenital-luetischen Individuen gesehen hat, die er als der Endarteritis obliterans ähnlich beschreibt, haben wir niemals gefunden. Es sei hier auf die seinerzeit von Fischl beschriebene Besonderheit der Gefäße im ersten Kindesalter hingewiesen. Nach diesem Autor charakterisiert sich die Gefäßwand in diesem Alter durch eine erheblichere Dicke, ein Merkmal, das in späterer Zeit wieder verloren geht. Sowohl dieser Umstand als auch das Vorhandensein von Kontraktionszuständen im Bereiche der glatten Muskulatur der Gefäßwand muß große Vorsicht bei der Beurteilung, ob es sich um pathologisch veränderte Gefäße handelt, nahelegen. So mußten wir auch Bilder Hochsingers, bei denen es sich unter anderem auch um endarteriitisch veränderte Gefäße handelt, anders beurteilen, indem uns die dort als pathologisch veränderte Gefäßwand öfters nicht von der Norm abweichend erschien.

Was nun das Vorkommen von Spirochäten in den Nieren der in dieser Gruppe zusammengefaßten Fälle anlangt, so wäre darüber folgendes zu berichten. Ihre Darstellung ist dort, wo sie vorhanden sind, sehr einfach. Regelmäßig wandten wir die Methode nach Levaditi an. Es schadet auch nicht, wenn die zu dieser Imprägnation verwendeten Organstücke längere Zeit in Alkohol verweilen, wie wir dies selbst feststellen konnten, und wie es auch von Gierke beschrieben wurde. 
In zwei Fällen (Nr. 1 und 4) waren Spirochäten reichlich vorhanden. Es wurde schon darauf hingewiesen, daß sie nicht gleichmäßig über die einzelnen Gewebe der Nieren verteilt waren. Vor allem war es auffallend, daß sie, während sie reichlich in den Kanälchen und im Interstitium vorhanden waren, die Glomeruli zum größten Teile frei ließen, und daß selbst in den Glomerulis, in denen sie gefunden wurden, nur verhältnismäßig wenige Exemplare vorhanden waren. Die Spirochaeta. pallida ist nicht der einzige Mikroorganismus, der das Bild einer derartigen Verteilung bei reichlichem Vorhandensein in den Nieren bietet. Muscatello und Ottaviano berichteten darüber folgendes. Sie injizierten Kaninchenaufschwemmungen von Staphylokokken in die Ohrvene und fanden bei der Untersuchung der Nieren in den Schlingen der Glomeruli sehr spärliche, zumeist aber gar keine Kokken. Später konnte ich gelegentlich einer Untersuchung der durch den Streptococcus mucosus hervorgerufenen pathologischen Veränderungen für letztgenannte Kokkenart dieselbe Figenschaft bezüglich ihrer Verteilung in den Nieren bei massenhafter Utberschwemmung des Organs durch diese Kokken feststellen. Für diese besondere Art der Verteilung der Mikroorganismen in den Nieren dürfte folgende schon seinerzeit für die Mucosuskokken gegebene Erklärung am naheliegendsten sein, die auch für die hier besprochenen Fälle Verwendung finden könnte. Sie dürfte auf einer Besonderheit des Verlaufes der arteriellen Gefäße in den Nieren beruhen. Im Nierenbecken teilt sich die Nierenarterie in eine Anzahl von Ästen, die in die zwischen den Pyramiden gelegene Corticalsubstanz treten. Dieselben verlaufen von hier aus nach wiederholter Teilung an der Grenze zwischen Rinde und Mark. Von diesen Verästelungen gehen dann die Arteriolae rectae und die Vasa afferentia der Glomeruli ab. Nach Henle übertreffen nun die Arteriolae rectạe, deren Durchmesser $0,02-0,03 \mathrm{~mm}$ beträgt, die Gefäße, von denen sie abgehen, um das Zwei- bis Dreifache ihres Durchmessers. Dieser Durchmesser nimmt auch im weiteren Verlaufe, wenn sich die Gefäße auf ihrem Wege unter spitzem Winkel gabelförmig teilen, nur in ganz geringem Maße ab. Infolge dieser Eigentümlichkeit halten wir es für sehr wahrscheinlich, daß die größere Anzahl der Mikroorganismen, die in den Gefäßen der Niere zirkulieren, in diese Gefäße eingeschwemmt wird. Was den Umstand betrifft, daß die Spirochäten, wenn auch selten, so doch immerhin öfters als seinerzeit die Kokken, in den Glomerulis zu finden waren, so dürfte das höchstwahrscheinlich der Bewegungsfähigkeit der Spirochäten zuzuschreiben sein. Diese Eigenschaft fehlt der Gruppe des Streptokokkus. Wenn wir nun der Ursache nachgehen wollen, warum in zwei der untersuchten Fälle Spirochäten reichlich gefunden wurden, in zwei Fällen vollkommen fehlten, so müssen wir folgende Umstände in Erwägung ziehen. Es soll noch bemerkt werden, 
Beitrag zur Kenntnis der Nierenerkrankungen bei kongenitaler Lues. 263

daß wir uns immer derselben Methode zur Darstellung der Mikroorganismen bedienten, die uns bei langer Anwendungsdauer in den verschiedenen Organen sichere Resultate gab. Bei genauerer Betrachtung zeigen die Fälle, in denen keine Spirochäten gefunden wurden, Unterschiede den Fällen gegenüber, in denen ein positiver Spirochätenbefund erhoben werden konnte. Während uns die Obduktionsprotokolle der Fälle Nr. 1 und 4 darüber belehren, daß in keinem derselben an irgendeiner Stelle des Körpers gummöse Veränderungen gefunden wurden, finden wir in den beiden anderen Fällen jedesmal Gummositäten, und zwar immer Gummen der Lunge verzeichnet. Auch die mikroskopischen Veränderungen der Nieren sind in den Fällen Nr. 1 und 4 von denen in den Fällen Nr. 2 und 3 verschieden. Es wurde darauf hingewiesen, daß in zwei Fällen Herde gefunden wurden, die durch die Art der sie bildenden Zellen, durch die schärfere Abgrenzung und durch die weiter fortgeschrittenen Gefäßveränderungen als ältere gegenüber den in den zwei anderen Fällen vorhandenen jüngeren Entzündungsprozessen angesehen werden müssen. In den Nieren nun, in denen jene älteren Herde vorhanden waren, fehlten auch die Spirochäten vollkommen, während sie in den beiden anderen Fällen reichlich gefunden wurden. Befunde, die mit den hier gemachten in Einklang zu bringen sind, sind in der Literatur vorhanden. So fand Versé bei sechs Fällen von kongenitaler Lues Spirochäten am zahlreichsten in unveränderten Organen und vermißte sie in den am schwersten geschädigten. Versé nimmt an, daß die Spirochäten auf dem Blutwege in das Stützgewebe und von hier aus in das Parenchym kommen. Dort, wo sie sich ansiedeln, sollten sie eine Reaktion des Gewebes auslösen, durch die sie selbst bis zum vollständigen Verschwinden vernichtet würden.

Auf Grund des Vorhergehenden möchten wir im Gegensatze zu Karvonen und seinen Anhängern bemerken, daß die Diagnose der kongenitalen Nierensyphilis beim Neugeborenen nicht auf Grund des Vorhandenseins irgendwelcher sogenannter Entwicklungshemmungen, sondern nur auf Grund der von uns regelmäßig gefundenen interstitiellen herdförmigen Entzündungsprozesse und deutlichen Alterationen der Tunica externa der Gefäßhaut gemacht werden kann. Diese Herde sind so charakteristisch, daß es nicht noch anderer luetischer Symptome, wie dies Hahn verlangt, zur Festigung der Diagnose bedart. Dazu kann eine eventuelle Bestätigung durch einen positiven Spirochätennachweis hinzukommen. Doch ist ein Fehlen von Spirochäten bei vorhandenen entzündlichen interstitiellen Herden von der hier beschriebenen Art nicht gegen die Diagnose einer luetischen Nierenaffektion zu verwerten.

\section{Gruppe: II.}

Diese Gruppe umfaßt solche Fälle, die schon einige Wochen oder Monate alt in die Klinik eingebracht wurden, oft floride Erscheinungen 
einer Lues aufwiesen, und die infolge ihres frühzeitig eingetretenen Todes entweder überhaupt nicht oder nur ganz kurze Zeit und daher ungenügend antiluetisch behandelt wurden.

Fall 1. R. E., geb. 9. X. 1921. Auf die Klinik aufgenommen 28. XI. 1921. Mutter vor 3 Jahren einer Genitalaffektion wegen mit Quecksilber und Salvarsan behandelt, gegenwärtig Papeln am Genitale. Vor 1 Jahr gebar sie ein totes Kind. WaR. positiv.

Kind: WaR positiv. In den ersten Lebenstagen Blasenaussohlag an beiden Soblen, später auch an anderen Stellen. Aufnahmsbefund: Uther die ganze Körperoberfläche zahlreiche teilweise frische, teilweise bereits abgeblaßte Papeln. verstreut: Eitrig-blutiger Schnupfen. Milz und Leber nicht vergrößert. Ödem des Genitales und der Fußrücken. Harn: Eiweiß positiv. Sediment: Vereinzelte Leukocyten. Gewicht $2200 \mathrm{~g}$. - 29. XI. erste Schmierkur. 2. XII. Neosalvarsan 0,03 intramuskulär. Stärkere Ödeme, Eiweiß positiv. Sediment: Leukocyten und vereinzelte Erythrocyten. 4. XII. Exanthem im Rückgange. 5. XII. Kind verfällt zusehends. 6. XII. Exitus letalis.

Obduktionsprotokoll: Einige weiße, pneumonische Herde im rechten Oberlappen. Luetisches Exanthem der Haut. Hochgradige Anämie und Ödem des Gehirns. Knorpelknochengrenze normal. Nieren: makroskopisch Anämie.

Mikroskopisch: An keiner Stelle ist mehr eine neogene Zone vorhanden. Uberall sind die Glomeruli durch eine Schicht gewundener Nierenkanälchen von der Kapsel getrennt. Das Bindegewebe ist im Bereiche der ganzen Niere in normaler Menge vorhanden, die Glomeruli zeigen keinerlei Anzeichen einer Entzündung. Dagegen sind die Kanälchenepithelien gequollen. Ofters zeigt ihr Plasma blasiges Aussehen. Die Kerne haben stellenweise an ihrer Tinktionsfähigkeit Einbuße erlitten, die Gefäße der Rinde sind nur in geringem Grade, und zwar nicht einmal regelmäßig, im Bereiche ihrer Tunica externa verändert. Die Gefäße des Markes zeigen keine Abnormitäten. Auch in diesem Falle sind interstitielle infiltrierende Prozesse vorhanden. Sie erreichen aber nirgendwo bedeutende Ausdehnung. Ihre Verbreitung ist eine mehr diffuse. Dadurch läßt sich eine schärfere $\mathbf{A b}$ grenzung gegen das gesunde Gewebe manchmal nur schwer erkennen. Am deutliohsten sind diese Infiltrate auoh hier um Gefäße vorhanden. Sie bestehen aus Lymphocyten und aus Zellen mit größerem Plasmahof, unter denen einzelne Plasmazellen gut zu erkennen sind. Daneben findet sich junges Bindegewebe. Cystische Gebilde in der peripheren Rindenzone sind nicht vorhanden. In den nach Levaditi behandelten Präparaten wurden keine Spirochäten gefunden.

Fall 2. H. V., geb. 18. VII. 1921, auf die Klinik aufgenommen 8. X. 1921. Mutter angeblich nie Erscheinungen. von Lues gehabt. März 1920 gebar sie ein totes Kind.

Kind: WaR. positiv. In der 6. Lebenswoche Ausschlag am ganzen Körper. Aufnahmestatus: Gewicht $2920 \mathrm{~g}$, Länge $51 \mathrm{~cm}$. Palmae und Plantae glänzend und schuppend. Zahlreiche Papeln über den Stamm verteilt. Einzelne Papeln auch im Gesicht. Nasenwurzel sattelförmig eingezogen. Typische Ragaden an der Lippenhautgrenze. Axillare und inguinale Lymphknoten bohnengroB und hart. Leber und Milz vergröBert. Deutliches Schniefen. Harn: EiweiB $\theta$, keine Formelemente. 2. X. Beginn der ersten Schmierkur. 13. X. Harn: Eiweiß 0. 14. X. Papeln blassen ab. 15. X. Temperatur bis $38^{\circ}$. Harn: Eiweiß positiv. Sediment: Zahlreiche Leukocyten, vereinzelte Erythrocyten, keine Zylinder. Neosalvarsan 0,03. 19. X. Papeln fast abgeheilt. Temperatur normal. - Zweite Schmierkur. Harnbefund unverändert. 26. X. Neuerlicher Fieberanstieg bis $38^{\circ}$. 27. X. Bronchopneumonische Herde im rechten Oberlappen, Kind verfällt 
zusehends. Im Harn stets der gleiche Befund. Andauerndes Fieber. 3. XI. Exitus letalis.

Obduktionsbefund: Fast ausgeheilte Ragaden an den Lippen und typische Sattelnase. Osteochondritis luetica. Ein erbsengroßes Gumma der rechten Femurdiaphyse. Degeneration der Leber. Atrophie der Darmschleimhaut bei allgemeiner Atrophie. Cystitis und Pyelitis. Ödem des Gehirns. Nieren: makroskopisch zahlreiche über die ganze Oberfläche verstreute gelbliche bis mohnkorngroße Erhebungen.

Mikroskopisch: An keiner Stelle der Nierenrinde kann das Vorhandensein einer neogenen Zone beobachtet werden. Eine Schicht gewundener Kanälchen trennt die oberste Reihe der Glomeruli von der Nierenkapsel. Das Bindegewebe ist, wo es keine entzündlichen Infiltrate aufweist, nicht vermehrt. Die Glomeruli zeigen keine Merkmale entzündlicher Veränderungen. In den Kanälchen, deren Epithelbelag deutliche Zeichen von Degeneration aufweist, finden sich massenhafte Leukocytenzylinder. Die Veränderungen ähneln denen der Nieren der Fälle 2 und 3 der ersten Gruppe. Es sind hier meist circumscripte Herde vorhanden, die deutlich Gefäße umlagern. Die Gefäße zeigen mehr oder weniger starke Auflockerung bzw. Infiltration ihrer Tunica externa. Die infiltrierten Herde bestehen neben einer geringen Anzahl von Lymphocyten aus plasmareicheren Zellen, unter denen die Plasmazellen einen gewissen Anteil ausmachen sowie auch aus faserigem Bindegewebselementen. Das Ausmaß dieser Herde erreicht oft das der größten Herde der Nieren der Fälle in Gruppe 1. Neben diesen Herden finden sich im Gebiete der äußersten Rindenzone solche, die fast ausschließlich aus faserigen kernreichem Bindegewebe bestehen und nur spärlich von Lymphocyten durchsetzt sind. Diese sind scharf von den übrigen Gewebspartien abgesetzt. Im Zentrum dieser Herde finden sich oft mit kubischem Nierenepithel ausgelegte und mit Flüssigkeit erfüllte Cystchen. Spirochäten wurden in den daraufhin gefärbten Präparaten nicht gefunden.

Fall 3. F. M., geb. 21. III. 1921, auf die Klinik aufgenommen 31. III. 1921. Mutter: WaR. positiv. Reflektorische Pupillenstarre.

Kind: $1300 \mathrm{~g}$ schwer, $40 \mathrm{~cm}$ lang. Aufnahmsbefund: WaR. negativ. Hautdecken gelb verfärbt. Fontanelle eingesunken. Nabelwunde abgeheilt. Soor der Zunge. Harn $\theta$. 4. IV. $1200 \mathrm{~g}$. Geyichtsstillstand bis zum. 15. IV. An diesem Tage zahlreiche flüssige Stühle. Harn: Eiweiß positiv. Sediment: zahlreiche Leukocyten. Kind verfällt rapid. 16. IV. Exitus letalis.

Obduktionsprotokoll: Katarrhalische Bronchitis mit Atelektasen in allen Lungenlappen. Hämatome in beiden Nebennieren. Cystitis und Pyelitis. Allgemeine Atrophie. Geringgradige Osteochondritis luetica. Atrophie der Nieren.

Mikroskopisch sind zweierlei pathologische Prozesse vorhanden. Die Veränderungen des Parenchyms erstrecken sich auf die Tubuli recti und auf die Sammelkanälchen. Die Kanälchen sind erweitert, ihr Epithelbelag gequollen. Stellenweise sind Lücken im Epithelbelag vorhanden. Das Innere der Kanälchen ist durch zahlreiche aus Epithelien und Leukocyten zusammengesetzte Zylinder erfüllt. Die Glomeruli zeigen keine entzündlichen Erscheinungen. Pseudoglomeruli sind an keiner Stelle vorhanden. Das interstitielle Bindegewebe präsentiert sich in normaler Menge. Pathologische Veränderungen des Interstitiums sind nur stellenweise nachweisbar. Sie kennzeichnen sich durch geringe perivasculäre Infiltration. Diese Infiltráte sind zum großen Teil aus Lymphocyten zusammengesetzt. Die Gefäße lassen keine Veränderungen erkennen. In den Levaditi-Präparaten wurden keine Spirochäten gefunden. 


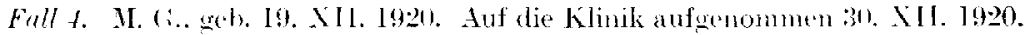

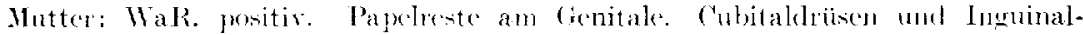
driesen hate und reryigiliert.

Kind: Aufnahmolefund: 2300 or schwer, 48 ('m lang. Wak negatir. Harn: 0 .

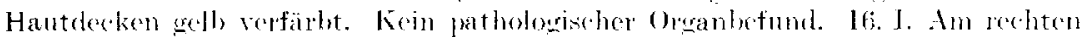

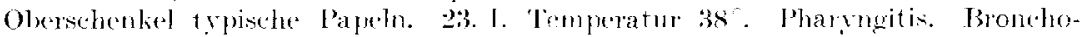
pneumonie im rechten Otserlappen. 2ofs, I. Exitus letalis.

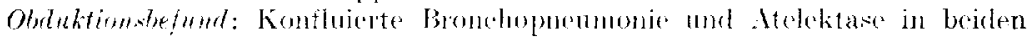
Oberlappere. Multiple hanfkorngrober Blutungen in der Leber. Degeneration des Herzmukels. Knowenknorpedrenze mmal. Niere: nakroskopisch ohne Veränderungeren.

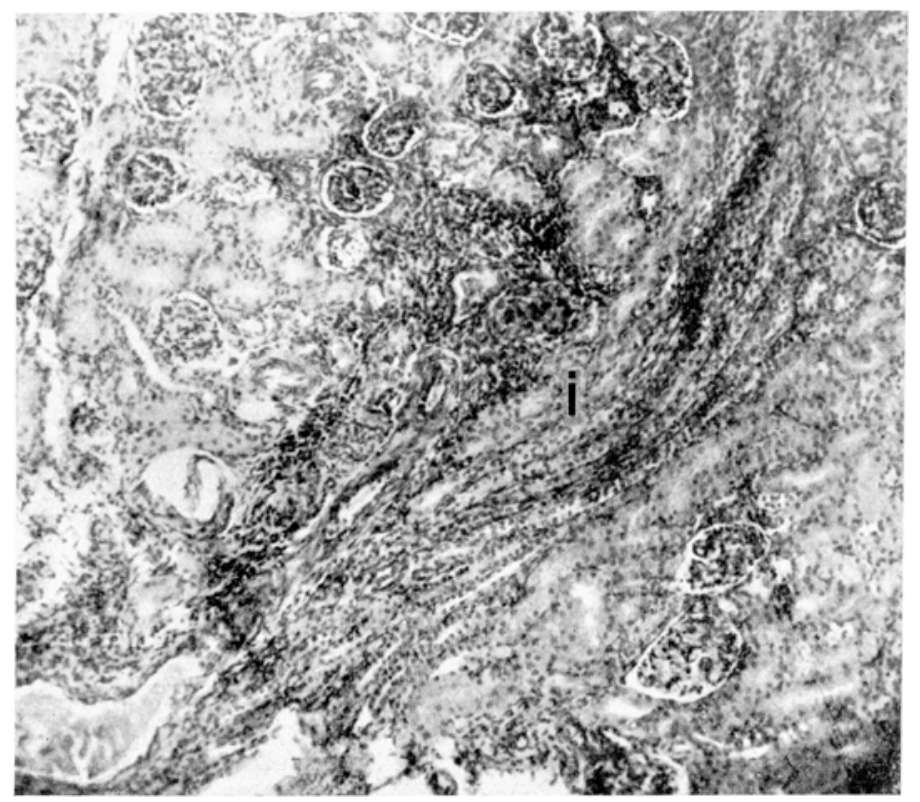

Abb. $\therefore$.

Miliroskopioch erwejet sich clas Parenchym bis auf geringeradige Degenerationserscheinungen volkommen normal. Eine neogene zonc ist nirgendwo rorhanden. (ystische Bildungen fehlen in Bereiche der Nierenrinde. Das Binde. gew he ist bis auf wenige Stellen nicht vermehrt. An vereinzelten stellen finden sich infiltrierte Hercle, die aröbre Ausdehnumg encichten. Dic diese Herde bildenden Zellen waren neben Lrmphoevten Plasmazellen und Epitheloidzellen sowie binderewebige Elemente. Die Herde waren so grol, dab sie in 18 -20 aufeinanderfolgenden terienschnitten von s:" Dicke zu rerfolgen wasen (Abb. 5, i). Stellenwoire fanden sich in ihnen kleinste nekrotische Partien. Die (iofäbe im Be. reiche dieser Herde zeigrten die schon geschidderten Veränderungen. In den nach Levediti gefärbten Präparaten konnten sipirochäten nicht gefunden werden.

Full j. V. I., geb. 20. IX. 1919. aufgenommen 21. 1X. 1919. Mutter: MaR. positiv, keine sicheren Zeichen von Lues. Kind: WaR. positiv. Aufnahmshefund: $1950 \mathrm{~g}$ schwer, $43 \mathrm{~cm}$ lang. Haut braunrötlich, Nabel haftend, 4. X. Kind ist 


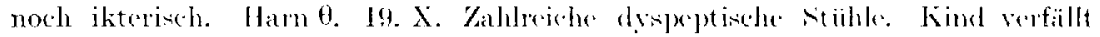
darumter ansehendis. 31. X. Exitus letalis.

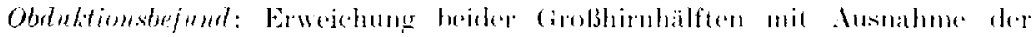

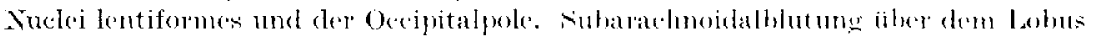

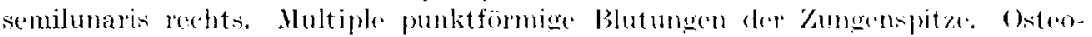

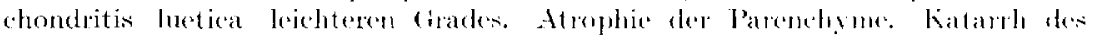
Magens und des Diekdarms. Witrige rechtssedige otitis media (hanzedformige

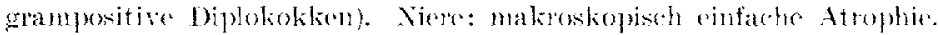

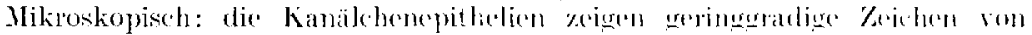

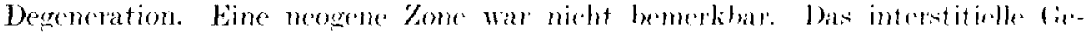

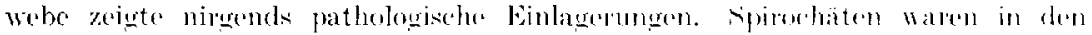
silberpriparaten nirgends zu findern.

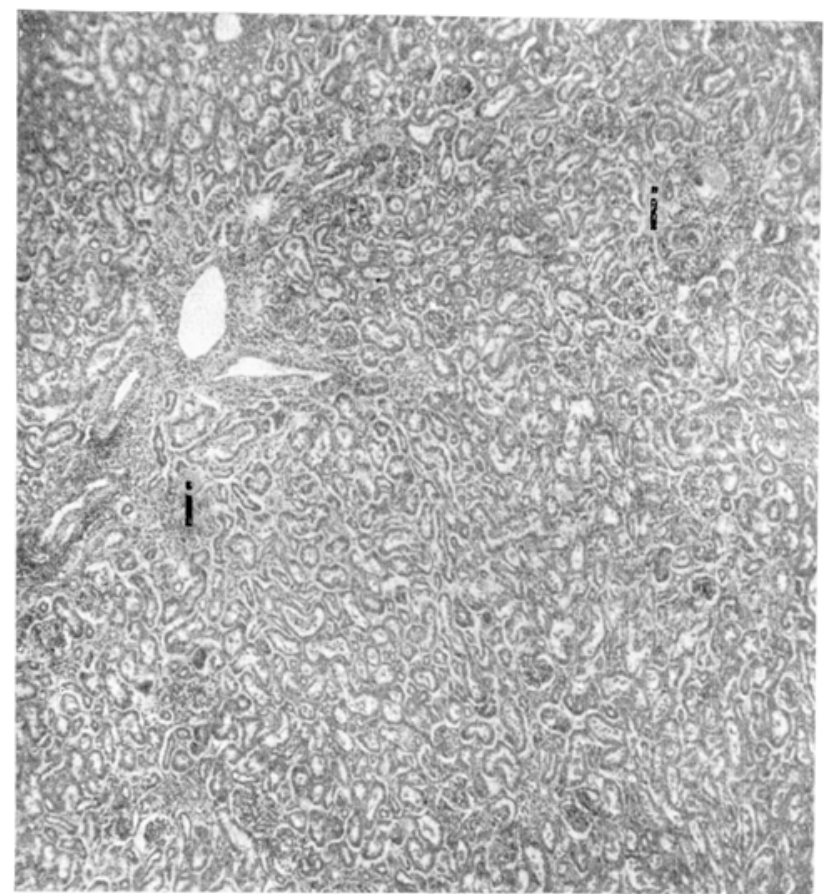

Ibl. 6.

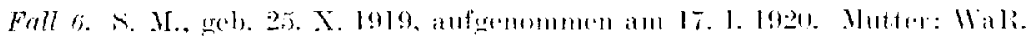
positiv. Kind: Aufnahmshefumel. Hautdecken fahlowll, Conjumetival gall,

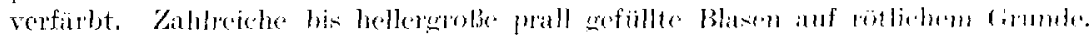

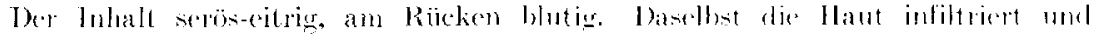

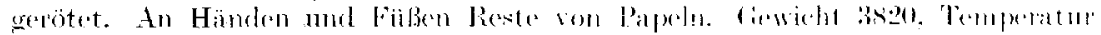

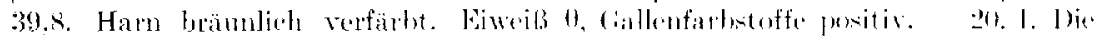

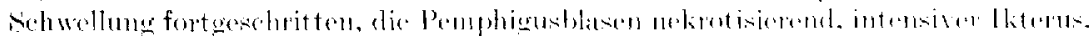

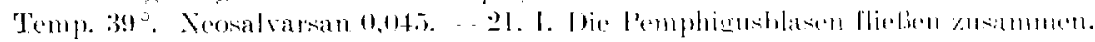
die Hant exulateriert. 2.). I. Fxitus letatio.

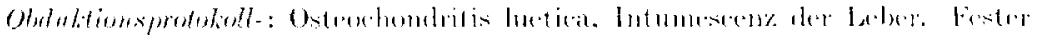

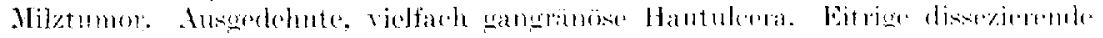


Periostitis und Perichondritis der 2. und 3. Rippe rechts. Multiple Abscesse in den Lungen. Beiderseitige fibrinös-eitrig hämorrhagische Pleuritis. Kompressionsatelektasen beider Unterlappen. Allgemeiner Ikterus. Degeneration der Parenchyme. Ödem des Gehirnes. Im Eiter der Abscesse Staphylococcus pyogenes aureus. Niere: makroskopisch Degeneration.

Mikroskopisch: Die Epithelien der Harnkanälchen, ebenso die Epithelien der Glomerulusschlingen sind gequollen. Das Bindegewebe ist in breiterer Schicht vorhanden. Die in demselben verlaufenden Arterien zeigen öfters eine Infiltration der äußeren Partien der Tunica externa. Das interstitielle Bindegewebe ist von zahlreichen kleinen, nicht scharf abgegrenzten, manchmal miteinander konfluierenden Herden durchsetzt (Abb. 6, I, S. 267). Sie bestehen weniger aus Lymphocyten als aus plasmareicheren Zellen, unter denen zahlreiche Plasmazellen bemerkbar sind. Daneben ist Bindegewebe vorhanden. In der Rinde sind keine cystischen Bildungen vorhanden.

Anschließend an diese Fälle sei hier noch über einige andere berichtet, die langdauernde antiluetische Kuren an unserer Klinik durchgemacht haben.

Fall 1. T. A., geb. 12. XII. 1920, auf die Klinik aufgenommen 22. XII. 1920. Mutter: WaR. positiv. Ausgebreitetes Leukoderm. Erstes Kind an Masern gestorben, wurde anderswo antiluetisch behandelt. Kind: Aufnahmsbefund: $2820 \mathrm{~g}$ schwer, 45,5 cm lang. Nabelwunde im Eintrocknen begiffen. Keine Symptome von Lues. WaR. positiv, - 28. XII. Akute Dyspepsie. Harn $\theta$. - 24. I. Bronchopneumonie. Temperatur bis $38,3^{\circ}$. Harn: Eiweiß positiv. - 1. II. Lungenbefund im Rückgange. Leichte Temperatursteigerungen. Harn $\theta$. -3 . II. Eine kleine Papel neben dem Nabel; erste Schmierkur. - 5. II. Harn: Eiweiß positiv. Sediment: zahlreiche Leukocyten und Epithelien der abführenden Harnwege. Temp. 38, $8^{\circ}$ - 16. II. In der Umgebung des Afters drei frische Papeln. Harn $\theta$. Bis zum 13. III. vier Schmierkuren und zwei Neosalvarsaninjektionen 0,03. Rhagaden an der Unterlippe trocken, die Papeln kaum sichtbar. Kind hypertonisch. - 7. III. Pharyngitis. Temp. 38 . - 29. III. Seit einigen Tagen schlechte Stühle. Kind nimmt konstant ab. Harn: Eiweiß positiv. Sediment: zahlreiche Leukocyten. 27. III. Kind verfält. - 2. IV. Bronohopneumonie. Exitus letalis,

Obduktionsprotokoll: Strahlenförmige narbige Einziehungen an der Übergangsstelle vom Lippenrot zur. Haut. Chroniseher Milztumor. Multiple Defekte der Tabula int. des Schädeldaches über der linken Großhirnhälfte. Chronische Enterocolitis mit Pigmentierung der Follikel im ganzen Dickdarm. Fettige Degeneration der Leber. Allgemeine Atrophie. Knorpelknochengrenze normal. Nieren: makroskopisch degeneriert.

Mikroskopisch: Eine neogene Zone ist nirgends vorhanden. In der Rindenschicht sind vereinzelte, sekreterfüllte, cystische Hohlräume vorhanden. An den Glomeruli sind keinerlei entzündliche Erscheinungen bemerkbar. Die Epithelien der Kanälchen zeigen gequollenes Protoplasma. Das Bindegewebe erscheint an vereinzelten Stellen hyperplastisch. Von einer Infiltration an diesen Stellen sind nur geringe Spuren bemerkbar. Spirochäten wurden in keinem der untersuchten Präparate gefunden.

Fall 2. K. R., geb. 6. V. 1920, aufgenommen 1. VI. 1920. Mutter: WàR. negativ. Allgemeine Drüsenschwellung. Kind: Aufnahmsbefund: Gewicht $2550 \mathrm{~g}$, $47 \mathrm{~cm}$ lang. Schnupfen. Teilweise blutig-belegte und wunde Mundwinkel. Am Kinn, an den Händen und Füßen typische luetische Papeln. Ausgedehntes papulöses Exanthem am Gesäß. Milz nicht tastbar. WaR. negativ. Harn $\theta$. Vom 
1. VI. bis 23. VII sieben Schmierkuren. - 4. VI. Papeln teilweise eingetrocknet. 9. VI. Alle Papeln im Eintrocknen. Harn $\theta .-13$. VI. Durchfall. Harn: Eiweiß positiv. Sediment: zahlreiche Leukocyten. 8.'VIII. Entlassen. - 11. XI. Wiederaufnahme. An den Händen und unteren Extremitäten bis hellergroße, kupferrote schillernde Flecken. Milz- und Lebertumor. - 12. XI. bis 5. XII. Drei Schmierkuren und zwei Neosalvarsaninjektionen 0,04 u. 0,05. Frische Papeln über dem rechten Auge. Harn $\theta$. - 20. XI. Milz und Leber kleiner. - 14. XII. Große Fontanelle stark vorgewölbt. Im Lumbalpunktate der Befund einer eitrigen Meningitis. - 15. XII. Exitus letalis.

Obduktionsprotokoll: Katarrhalische Bronchitis, frische lobulär-pneumonische Herde in beiden Lungen. Eitrige Meningitis der Konvexität des Großhirns. Fettige Degeneration der Leber. Chronischer Milztumor. Knorpelknochengrenze normal. Niere: makroskopisch fettige Degeneration.

Mikroskopisch: Die Epithelien der Kanälchen mäßig verfettet, die Glomeruli normal. Keine pathologischen Veränderungen im Interstitium.

Fall 3. T. M., geb. 1. VIII. 1921. Auf die Klinik aufgenommen 10. VIII. 192I. Mutter während der Schwangerschaft mit Quecksilber behandelt. WaR. positiv. Kind: Aufnahmsbefund. $2500 \mathrm{~g}$ schwer, $47 \mathrm{~cm}$ lang. An den Füßen papulomaculöses Exanthem. 12. VIII. bis 17. XI. Zwölf Schmierkuren und fünf Neosalvarsaninjektionen 0,03 . Harn $\theta$. - 17. VIII. Harn $\theta$. - 19. VIII. Zahlreiche flüssige Stühle. Harn: Eiweiß positiv. Sediment: Plattenepithel und vereinzelte Leukocyten. - 26. VIII. Harn $\theta$. Exanthem geschwunden. - 25. X. Harn $\theta$. Leber und Milz tastbar. - 16. I. Gewicht 4000 g. - 17. I. Grippe. Temp. 38 ${ }^{\circ}$. 23. I. Temp. $38,5^{\circ}$. Harn: Eiweiß positiv. Sediment: zahlreiche Leukocyten. 30. I. Harn 0. - 31. I. Kollaps, Exitus letalis.

Obduktionsbefund: Chronisch schleimige Colitis mit Pigmentierung der Follikel im Dickdarm. Atrophie der Leber. Hochgradiges Ödem und Hyperämie des Gehirns. Ecchymosen in den Pleuren und im Epikard. Chronischer Milztumor mäßigen Grades. Knorpelknochengrenzen normal. Anämie und Degeneration.

Mikroskopisch: Die Glomeruli zeigen keinerlei Veränderungen. Das Epithel der Harnkanälchen ist degeneriert. In den Kanälchen finden sich Zylinder. Das interstitielle Bindegewebe ist nicht vermehrt und zeigt keine pathologischen Einlagerungen. In den nach Levaditi behandelten Präparaten wurden keine Spirochäten gefunden.

Betrachten wir nun die in den beiden Gruppen 2 und 3 beschriebenen Fälle zuerst bezüglich ihres klinischen Verlaufes, so läßt sich darüber folgendes berichten. In den Harnen vieler dieser Kinder ließen sich während der Zeit ihres Aufenthaltes an der Klinik teils vorübergehend, selten bis zum letalen Exitus andauernd, Eiweiß und Formelemente, wie Zylinder, Epithelien, Leukocyten und Erythrocyten, nachweisen. Nur die Fälle Nr. 4, 5 und 6 der 2. Gruppe zeigten niemals während der Beobachtungszeit einen positiven Harnbefund. Von diesen Fällen bot der Fall Nr. 4 geringe, Fall Nr. 5 keine und Fall Nr. 6 ausgedehnte mikroskopisch nachweisbare Veränderungen des Interstitiums. Von den übrigen Fällen, die einen positiven Harnbefund aufwiesen, konnte dieser nur einmal in kausalen Zusammenhang mit der Lues gebracht werden. Vor allem schiedęn Fall 2 und 3 der 2. Gruppe aus, bei denen neben einem stark positiven Eiweißbefunde das Vorhandensein von 
zahlreichen Leukocyten und von Epithelien der abführenden Harnwege in beiden, überdies das Auftreten von Erythrocyten im zweiten Falle nachgewiesen werden konnte. Bei diesen beiden Fällen wurde autoptisch die Diagnose einer Cystopyelitis bestätigt. Was die restlichen Fälle, die einen positiven Harnbefund aufwiesen, anlangt, so stand bei ihnen dieser Befund stets mit einer Ernährungsstörung oder mit einem fieberhaften Infekte in Zusammenhang. Bei Fall 1 der 3. Gruppe trat zu Beginn der ersten Quecksilberkur inmitten der Eruption eines papulösen Exanthems Eiweiß im Harn auf. Das gleichzeitige Erscheinen von zahlreichen Leukocyten und Epithelien, sowie der Anstieg der Temperatur bewiesen aber, daß dieser Harnbefund nicht mit der bestehenden Lues in Zusammenhang zu bringen, sondern als Zeichen einer Entzündung der Blase aufzufassen wäre, wie sie bei kongenital-luetischen Säuglingen, die sich ja meist gegen alle Arten von Infektionsprozessen recht widerstandslos erweisen, keine Seltenheit ist. Einzig und allein der positive Harnbefund beim Falle Nr. 1 der 2. Gruppe konnte mit der Lues in Zusammenhang gebracht werden. Er zeigte bei der Aufnahme auf die Klinik neben dem Bestehen eines ausgedehnten papulösen Exanthems Eiweiß und wenige Leukocyten im Harn sowie ausgebreitete Ödeme.

Einige Autoren geben an, daß, trotzdem verhältnismäßig häufig pathologische Befunde bei der anatomischen Untersuchung der Nieren kongenital-luetischer Kinder gemacht wurden, zeitlebens klinisch meist keine abnormen Harnbefunde zu erheben waren. Andere Autoren wiederum behaupten das Gegenteil. So fand z. B. Hecker unter seinen Fällen $50 \%$, an denen er die Symptome einer ,parenchymatösen Nephritis“" feststellen konnte. Hahn fand bei weitem keinen derartig hohen Prozentsatz von klinisch nachweisbaren Nierenveränderungen bei seinem Untersuchungsmaterial. Bei unserem Material konnten wir unter den in den Gruppen Nr. 2 und 3 zusammengestellten neun Fällen nur einen einzigen solchen finden (also nur in ca. $11 \%$ ), bei dem der vorhandene Harnbefund sowie die Ödeme auf die Lues zurückgeführt werden konnten. Diese Prozentzahl würde sich noch um ein gutes Stück weiter nach unten verschieben, wenn wir diejenigen Fälle, die nicht ad exitum kamen und die wir hier nicht mit aufgenommen haben, in die Statistik einbezogen hätten, zumal wir auch sowohl bewogen durch die Angaben in der Literatur, als auch durch den rasch ad Exitum führenden Verlauf dieses Falles der Meinung sind, daß die Gruppe von Fällen, in der das Parenchym der Nieren von einer luetischen Affektion ergriffen ist, einen besonders schweren Verlauf zeigt. Auch Hinzelmann gibt in seiner Arbeit über die Nierenerkrankungen bei kongenitaler Lues einen bedeutend kleineren Prozentsatz als Hecker an. Dabei gestatten die von diesem Autor oft allzu kurz mitgeteilten Krankengeschichten dem 
Leser nicht, sich selbst ein Urteil zu bilden, ob es sich in diesen Fällen immer um spezifisch luetische Nierenerkrankungen gehandelt hat. Lange fand unter den von ihm untersuchten Kindern in $11 \%$ der Fälle Zeichen einer Nierenerkrankung; von diesen $11 \%$ aber scheidet ungefähr die Hälfte als nicht spezifisch bedingt aus.

Was nun die Art und die Zahl der Nierenveränderungen bei kongenital-luetischen Säuglingen betrifft, so wäre folgendes darüber zu sagen. Unter den neun hier untersuchten Fällen zeigten sechs mikroskopisch wahrnehmbare Veränderungen. Davon entfielen fünf auf die nicht oder ungenügend behandelten und einer auf die behandelten Fälle. Negative Resultate ergaben ein Fall der Gruppe 2 und zwei Fälle der Gruppe 3. Makroskopisch sichtbare Prozesse kamen im ganzen nur in einem Falle der Gruppe 2 zur Beobachtung und dokumentierten sich daselbst in Gestalt von mohnkorngroßen Knötchen. Die mikroskopischen Nierenveränderungen bestanden auch in diesen beiden Gruppen in interstitiellen Infiltraten. Die mit einer einzigen Ausnahme vorhandenen unspezifischen Prozesse am Parenchym bestanden aus einer mehr oder weniger fortgeschrittenen Degeneration. Aus dem mikroskopischen Bilde allein konnte niemals die Entscheidung getroffen werden, ob es sich um einen spezifischen oder unspezifischen parenchymatösen Prozeß handelte. Es mußte immer der klinische Befund bei der Diagnosestellung zu Hilfe genommen werden. Die Infiltrate im Interstitium der Nieren der beiden zuletzt besprochenen Gruppen waren prinzipiell nicht von denen in Gruppe 1 verschieden. Mehr als die in Gruppe 1 beschriebenen differierten sie in ihrer Größe und Ausdehnung untereinander. Am zahlreichsten und auch am größten waren die Herde in Fall 2, Gruppe 2 vorhanden, der sich durch bereits makroskopisch sichtbare Veränderungen auszeichnete. Uberaus zahlreich, allerdings in kleinerem Umfange, waren sie in Fall 6, Gruppe 2 vorhanden. Vereinzelte, aber große Herde zeigte Fall 4, Gruppe 2. Die Veränderungen der Nieren in den Fällen 1 und 3 der 2. Gruppe bestanden in kleineren perivasculären Infiltraten. Was die Gewebselemente betrifft, aus denen sich diese Herde zusammensetzen, so unterschieden sie sich auch darin nicht von den Fällen der 1. Gruppe. In ihrem Aussehen näherten sie sich meistens den Fällen 2 und 3 dieser Gruppe. Neben einer geringen Anzahl von Lymphocyten waren mehr Plasmazellen und andere Rundzellen vorhanden. Im Vordergrunde standen die Plasmazellen im Fall 6 der 2. Gruppe. Eine größere Anzahl von Lymphocyten wiesen die Fälle Nr. 3 und 4 der Gruppe 2 auf. Auf Grund des histologischen Aufbaues dieser Herde ergab es sich, daß sie verschieden alt waren, zumeist aber als längere Zeit bestehend angesehen werden mußten. Eine einzige Ausnahme bildete der Fall 3, wo die lymphocytären Elemente den größten Teil der Infiltrate ausmachten. Hier 
hat es sich entweder um einen kürzer bestehenden Prozeß oder um eine Infektion mit einem verhältnismäßig weniger virulenten Spirochätenstamme gehandelt, der nicht die starke Gewebsreaktion, wie sie in den übrigen Fällen vorhanden war, bewirken konnte. Daher kann es mit Rücksicht auf das, was bereits bezüglich der Fälle der Gruppe 1 gesagt wurde, nicht auffallend sein, wenn in diesen Fällen hier niemals Spirochäten nachgewiesen werden konnten. An den Gefäßen fanden sich auch hier wiederum Veränderungen, wie sie bei den Fällen der Gruppe I beschrieben wurden.

Ergänzend sei noch hinzugefügt, daß Entwicklungshemmungen am glomerulären Apparate in keinem der Fälle der letzten zwei Gruppen konstatiert werden konnte. Auch das interstitielle Bindegewebe zeigte, abgesehen von denStellen, an denen Infiltrate vorhanden waren, keinerlei Hyperplasie. Kleine cystische Gebilde in der Rindenperipherie, die auch in normalen Nieren recht häufig zu finden sind, kamen bei unseren Fällen nicht häufiger als es der Norm entsprechen würde vor und waren, wo sie auftraten, auf die Größe und die Anzahl beschränkt, wie wir sie auch bei nicht luetischen Fällen zu sehen gewöhnt sind.

Wenn wir nun den Ablauf dieser herdförmigen Entzündungsprozesse betrachten, so müssen wir uns vor allem vor Augen halten, daß die Prozentzahl der nicht behandelten Fälle, welche Veränderungen im Interstitium aufweisen, gegenüber den behandelten eine bedeutend größere ist. Unter den erwähnten sechs nicht behandelten Fällen fand sich nur ein Fall, bei dem völlig intakte Nieren vorhanden waren, während unter den drei behandelten Fällen zweimal ein normaler Nierenbefund erhoben werden konnte. Außerdem wäre noch hervorzuheben, daß es unter den antiluetisch behandelten Fällen gerade der jüngste war, der als einziger einen positiven Nierenbefund aufwies.

Die hier beschriebenen Befunde im Interstitium der Nieren kongenital-luetischer Kinder ähneln sehr den Nierenveränderungen, die Volhard in der Gruppe der septischen interstitiellen Nephritis zusammengefaßt hat. Bei dieser fehlt in der Regel ein positiver Eiweißbefund sowie Formelemente im Harn. Ebenso fehlen auch ödeme. Diese Art von Nephritis kommt als reine interstitielle Form ohne entzündliche Veränderungen an den Glomerulis vor. Sie präsentiert sich in der Form verschieden großer lymphocytärer Infiltrate im interstitiellen Gewebe. Was die Ausheilungsmöglichkeit einer derartigen Nephritis betrifft, so meint Volhard, daß hier weniger der Zustand der Nieren, als vielmehr der Verlauf der Grundkrankheit ausschlaggebend ist. Auch bei der Lues dürfte unseres Erachtens der Nierenbefund mit der Schwere bzw. mit dem Zeitpunkte der stattgehabten Infektion in Zusammenhang stehen. Bei den Fällen, die wohl als schwerste anzusehen sind, nämlich bei denjenigen, die wir in Gruppe 1 zusammen- 
gefaßt haben, waren auch die Nierenveränderungen stets in weitestem Ausmaße vorhanden. $\mathrm{DaB}$ leichter verlaufende Fälle nicht immer eine Beteiligung der Nieren aufweisen müssen; beweist der Fall aus der Gruppe der unbehandelten Fälle, der frei von jeder pathologischen Erscheinung an den Nieren war. Da es ja eine bekannte Tatsache ist, daß luetische Infiltrate auf antisyphilitische Kuren hin sehr leicht zurückgehen können, so glauben wir mit Rücksicht auf die hier untersuchten Fälle, und zwar besonders hinsichtlich des Falles 1 der 3. Gruppe, bei dem sich die pathologischen Erscheinungen in den Nieren dahin deuten ließen, daß es sich um einen in Rückbildung befindlichen Prozeß handelte, daß die durch die Lues bedingten interstitiellen Niereninfiltrate rückbildungsfähig sind. Thre Prognose richtet sich also auch nach dem Verlaufe der sie verursachenden Allgemeinerkrankung. Der Untersucher, der die Niere eines kongenital-luetischen Kindes aus dem späteren Säuglingsalter oder aus einer späteren Altersperiode zur Ansicht bekommt, wird also, handelt es sich nicht um schwere frühere Nierenveränderungen die sichtbare dauernde Veränderungen zurücklassen, öfters nicht in der Lage scin zu entscheiden, ob das betreffende Organ seinerzeit pathologische Veränderungen aufgewiesen hat oder nicht.

Es wäre noch über das Verhältnis dieser interstitiellen Prozesse zur Schrumpfniere zu sprechen. Nur in den allerschwersten Fällen bei weitest ausgebreiteten Veränderungen im Interstitium bzw. an den dort verlaufenden Gefäßen, dürfte es zur Entstehung einer Schrumpfniere kommen. Daß dies so selten der Fall ist, dürfte darin seinen Grund haben, daß die betreffenden Früchte meist schon intrauterin oder nach kürzester Lebenszeit zugrunde gehen. Es erscheint uns möglich, daß antiluetische Kuren der Mutter, die erst zu einem verhältnismäßig späten Zeitpunkte im Verlaufe der Gravidität einsetzen, zur Entstehung einer Schrumpfniere insofern beitragen könnten, als in solchen Fällen die narbige Ausheilung schon vorhandener luetischer Produkte durch die Kur veranlaßt würde, wodurch es schon im allerfrühesten Säuglingsalter zum Auftreten einer Schrumpfniere kommen könnte.

In der Literatur sind nur vereinzelte Fälle von luetischer Schrumpfniere beschrieben. Wie viele davon auf interstitielle Prozesse, wie viele und welche von ihnen auf die noch zu besprechenden Vorgänge im Parenchym zurückzuführen sind, muß vorläufig noch in Frage gestellt bleiben.

Neben diesen eben erwähnten interstitiellen Prozessen wären noch jene, welche sich im Nierenparenchym abspielen, zu besprechen. Wie aus den wiedergegebenen Protokollen hervorgeht, sind sie unvergleichlich seltener als die interstitiellen Veränderungen. In der $\mathrm{Li}$ teratur liegen zumeist kasuistische Berichte über Fälle von sogenannter 
„parenchymatöser" und hämorrhagischer Nephritis vor. Bei kritischer Betrachtung derselben scheidet ein Teil derselben als Nierenerkrankungen, die nicht auf luetischer Basis beruhen, aus, weil es bei ihnen nicht sicher erscheint, ob nicht interkurrente Infektionen oder Ernährungsstörungen diese Nierenschädigungen bedingen konnten. So erweisen sich also unter den überaus zahlreichen untersuchten Fällen von kongenitaler Lues bei strenger Kritik nur wenige als wirklich luetisch-bedingte parenchymatöse Nierenaffektionen. In der Reihe der von uns untersuchten Fälle ist es auch nur ein einziger, bei dem der pathologische Prozeß des Parenchyms auf die bestehende Lues zurückgeführt werden kann (Gruppe 2, Nr. 1). Daś betreffende Kind zeigte Zeichen einer Nephrose im Sinne von Volhard. Es waren Ödeme vorhanden, der Harn enthielt Eiweiß, wenige Leukocyten und nach einer erfolgten Neosalvarsaninjektion ganz vereinzelte rote Blutkörperchen. Das stärkere Hervortreten des Harnbefundes nach dem Beginne der antiluetischen Kur ist wohl analog der Herxheimerschen Reaktion, die sich an dem luetischen Exanthem im Verlaufe antiluetischer Kuren feststellen läßt, zu deuten. Auch die mikroskopische Untersuchung der Nieren, bei der sich eine vorgeschrittene Degeneration der Nierenkanälchen ohne jede Beteiligung der Glomeruli zeigte, sprach für die Diagnose Nephrose. Der anatomische Befund in unserem Falle unterschied sich von dem Allgemeinbild, das Hahn von den luetischen Parenchymerkrankungen der Nieren entwirft. Nach den Beschreibungen des genannten Autors müßte es sich, da er auch über ein Befallensein der Glomeruli berichtet, um Nephritiden mit sekundärem nephrotischen Einschlag handeln. Daß die kongenitale Lues hin und wieder den Grund zum Entstehen einer Nephrose bildet erscheint nicht absonderlich, da ja das Vorhandensein von Nephrosen bei aquirierter Lues keineswegs zu den Seltenheiten zu rechnen ist und man überdies die Vorliebe der Nephrose für das jugendliche Alter kennt. Daß diese luetisch bedingten Nephrosen in ihrem weiteren Verlaufe zu einer Schrumpfniere führen können, halten wir, obzwar wir nie einen derartigen Fall beobachten konnten, für möglich, weil ja die Entstehung einer Schrumpfniere auf nephrotischer Basis sichergestellt ist. Zum Unterschied von der rasch vorübergehenden Nephrose bei aquirierter Lues scheint die Prognose bei den Nephrosen, die durch kongenitale Lues bedingt sind, ernster zu sein.

Zusammenfassend ließe sich also über die Nierenerkrankungen bei ererbter Syphilis im Säuglingsalter folgendes sagen. Diese Organe sind bei kongenitaler Lues sehr häufig, keineswegs aber regelmäßig ergriffen. In den von uns beobachteten Fällen konnten wir zwei verschiedene Formen von luetischen Affektionen der Nieren feststellen.

1. Die interstitielle Form, die prinzipiell der bei luetischen Neugeborenen gefundenen gleicht und die sowohl in ihrem anatomischen Aus- 
Beitrag zur Kenntnis der Nierenerkrankungen bei kongenitaler Lues. 275

sehen als auch in ihrem klinischen Verhalten der nach Volhard als septische interstitielle Herdnephritis bezeichneten Nierenerkrankung gleichzusetzen ist. Wie diese macht sie auch keine klinischen Erscheinungen und ist rückbildungsfähig. Sie ist fast immer erst durch mikroskopische Untersuchungen festzustellen.

2. Die Form, bei der das Parenchym der Nieren ergriffen ist, präsentiert sich sowohl was ihren klinischen Verlauf als auch was ihr anatomisches Bild anlangt, als Nephrose im Sinne von Volhard. Sie tritt, verglichen mit der zuerst genannten Affektion des Interstitiums, unvergleichlich seltener auf. Ihre Prognose scheint ernst zu sein. Zu ihrer Diagnose erscheint uns niemals der anatomische Befund allein ausreichend, sondern es muß immer der klinische Verlauf des Falles mit berïcksichtigt werden.

Glomerulonephritiden konnten wir bei kongenitaler Lues nicht beobachten. Wir sind weit davon entfernt die Möglichkeit ihres Auftretens bei dieser Krankheit in Abrede zu stellen. Doch dürften sie noch viel seltener als die beschriebenen nephrotischen Prozesse auftreten.

\section{Literaturverzeichnis.}

1) Aschoff, Lehrb. d. pathol. Anat., Jena 1921. - 2) Cassel, Berl. klin. Wochenschr. 41. 1904. - ${ }^{3}$ ) Ceelen, Virchows Arch. f. pathol. Anat. u. Physiol. 211. 4) Finkelstein, Lehrb. d. Säuglingskrankh. Berlin 1921. - ${ }^{5}$ ) Fischl, R., Arch. f. Kinderheilk. \%. 1887. - ${ }^{6}$ ) Frank, Frankfurt. Zeitschr. f. Pathol. 24. - ') Gierke, Münch. med. Wochenschr. 1906. - 8) Hahn, Zeitschr. f. Kinderheilk. Ref. 22. $\left.{ }^{9}\right)$ Hecker, Beitr. z. Hist. u. Pathol. d. cong. Syph, Naumburg 1898. - 10) Hecker, Jahrb. f. Kinderheilk. 5i. 1900. - 11) Hecker, Verhandl. d. Ges. f. Kinderheilk. 1901, Hamburg. - ${ }^{12}$ ) Hente, Handb. d. Anat. Braunschweig 1866. - ${ }^{13}$ ) Hinzelmann, Zeitschr. f. Kinderheilk. 9. - 14) Hochsinger, Stud. über hered. Syph., Wien 1898. - $\left.{ }^{15}\right)$ Hutinel und Hudelo, Arch. de méd. expérim. 2. 1890. 16). Karvonen, Die Nierensyphilis, Berlin 1901. - ${ }^{17}$ ) Lange, Jahrb. f. Kinderheilk. 90. - 18) Mracel,, Arch. f. Dermatol. u. Syphilis, Orig. 15. - 19) Muscatello und Ottaviano, Virchows Arch. f. pathol. Anat. u. Physiol. 166. - ${ }^{20}$ ) Riedel, zit. nach Stroebe. - 21) Spanudis, zit. nach Hochsinger. - ${ }^{22}$ ) Stoerck, Wiener klin. Wochen-, schr. 14. 1901. - ${ }^{23}$ ) Stroebe, Zentralbl. f. pathol. Anat. 2, 1891. - ${ }^{24}$ ) Toldt Sitzungsber. d. k. k. Akad. zu Wien 1874, III. Abt. - ${ }^{25}$ ) Versê, Med. Klin. 2. 28) Volhard, Die doppelseitigen hämatogenen Nierenerkrankungen. Berlin 1918.

II. Deutsche Universitätskinderklinik Prag. 